Check for updates

Cite this: RSC Adv., 2019, 9, 4113

Received 15th November 2018 Accepted 8th January 2019

DOI: $10.1039 / \mathrm{c} 8 \mathrm{ra} 09409 \mathrm{~h}$

rsc.li/rsc-advances

\section{Overview of sesquiterpenes and chromones of agarwood originating from four main species of the genus Aquilaria}

\author{
Mei Gao, ${ }^{a}$ Xiaomin Han, ${ }^{b}$ Ying Sun, ${ }^{a}$ Hongjiang Chen, ${ }^{c}$ Yun Yang, ${ }^{d}$ Yangyang Liu, ${ }^{d}$ \\ Hui Meng, ${ }^{d}$ Zhihui Gao, ${ }^{a}$ Yanhong Xu, ${ }^{a}$ Zheng Zhang (D) *a and Jianping Han*a
}

\begin{abstract}
The main chemical constituents of agarwood are sesquiterpenes and chromones, which can be divided into different categories depending on their molecular skeletons. Agarwoods are obtained from different plant species: Aquilaria sinensis, A. malaccensis, A. crassna, and A. subintegra. In this review, we systematically summarized the structures of 367 compounds isolated from agarwoods originating from four main species. We structurally classified all the components into 11 different types and summarized the number of compounds in each type. Different and identical components were obtained by enumerating the chemical compositions of the different species. Knowledge regarding the chemical constituents of agarwoods of different species will aid understanding of the chemical compositions of agarwoods and will subsequently identify similar compounds that can serve as standards for quality control to provide a reference for future studies on agarwoods from different species and to increase their usefulness.
\end{abstract}

\section{Introduction}

Agarwood is a resinous portion of Aquilaria trees, a genus belonging to the family Thymelaeaceae. Agarwoods have wide uses in traditional medicine, for example, as aphrodisiacs, sedatives, cardiotonics, and carminatives, as well as in the relief of gastric problems, coughs, rheumatism, and high fever. ${ }^{1}$ In addition, agarwoods are present in important spices and are also used as incense. Agarwood is known as 'chenxiang' in Chinese and 'aloeswood', 'agalloch', 'eaglewood', 'jinkoh', 'gaharu', and 'kanankoh' in other parts of the world. ${ }^{2}$

Approximately 15 species of Aquilaria are well known for their production of fragrant heartwood, also known as gaharu, aloeswood or agarwood. Wounding of the tree appears to be essential for the initiation of gaharu production, and fungal infection is likely to enhance the process. According to Eurlings et $a l .,{ }^{3}$ the following nine Aquilaria species produce gaharu: $A$. beccariana, A. crassna, A. filaria (Oken), A. hirta, A. khasiana, A. malaccensis, A. microcarpa, A. rostrata and $A$. sinensis; these are

${ }^{a}$ National Engineering Laboratory for Breeding of Endangered Medicinal Materials, Institute of Medicinal Plant Development, Chinese Academy of Medical Sciences, Peking Union Medical College, Malianwabei Road, Beijing 100193, P. R. China. E-mail: zhangzheng@implad.ac.cn; jphan@implad.ac.cn; Tel: +86-10-57833363 ${ }^{b}$ Tianjin University of Commerce, No. 409 Guangrong Road, Beichen District, Tianjin 300134, P. R. China

${ }^{c}$ Zhejiang Pharmaceutical College, Ningbo 315100, P. R. China

${ }^{d}$ Hainan Provincial Key Laboratory of Resources Conservation and Development of Southern Medicine, Hainan Branch, Institute of Medicinal Plant Development, Chinese Academy of Medical Sciences, Peking Union Medical College, Wanning 571533, P. R. China mainly sourced from India, Southeast Asia, Papua New Guinea, and China (chiefly in Hainan and Guangdong). ${ }^{3}$

As stated in reports, sesquiterpenoids and phenylethyl chromone derivatives are the principal compounds in the oleoresin of agarwood, which are mostly found in the species Aquilaria, A. malaccensis, A. agallocha, A. sinensis, and A. crassna., ${ }^{\mathbf{1 , 4}}$ In 2016, a review of agarwood noted A. agallocha Roxb. (endemic in India), of which the species name is unresolved (Table 1). ${ }^{5}$ The index of CITES species, ${ }^{6}$ Missouri Botanical Garden website, ${ }^{7}$ and the Ayurvedic and Unani Pharmacopoeias all list $A$. agallocha Roxb. as a synonym of A. malaccensis Lam. Since then, there have been other reports on this species. ${ }^{8-10}$ Therefore, this article will incorporate all the chemical constituents reviewed in A. agallocha into A. malaccensis. Other genera of the family Thymelaeaceae, such as Aetoxylon, Gyrinops, Phaleria, and Gonystylus, have also been reported to produce agarwoods. ${ }^{3}$ It has been reported that different countries have endemic species; for example, $A$. crassna principally grows in Indochina, A. malaccensis is an Indomalesian type found in Malaysia, Thailand and India, and A. sinensis is endemic in China. A. subintegra is principally found in Thailand. ${ }^{8}$

All nine of the known Aquilaria species can produce agarwood; however, it is not known which species are most productive. Studies have shown that specific species produce specific chemical components which are quite different from one another. ${ }^{11}$ Assessing the similarities and differences between these components is a good way to identify species, determine their quality and classify specific species. 
Table 1 Species in the genus Aquilaria (accepted names) (The Plant List, 2013)

\begin{tabular}{ll}
\hline Species & Authorship \\
\hline A. apiculata & Merr., 1922 \\
A. baillonii & Pierre ex Lecomte, 1915 \\
A. banaense & P. H. Hô, 1986 \\
A. banaensis & P. H. Hô, 1986 \\
A. beccariana & Tiegh., 1893 \\
A. brachyantha & (Merr.) Hallier L, 1922 \\
A. citrinicarpa & (Elmer) Hallier L, 1922 \\
A. crassna & Pierre ex Lecomte, 1915 \\
A. cumingiana & (Decne.) Ridl., 1901 \\
A. decemcostata & Hallier L, 1922 \\
A. filaria & (Oken) Merr., 1950 \\
A. hirta & Ridl., 1901 \\
A. khasiana & Hallier L, 1922 \\
A. malaccensis & Lam., 1783 \\
A. microcarpa & Baill., 1875 \\
A. parvifolia & (Quisumb) Ding Hou, 1960 \\
A. rostrata & Ridl., 1924 \\
A. rugosa & K. Le-Cong and Kessler, 2005 \\
A. sinensis & (Lour.) Spreng., 1825 \\
A. subintegra & Ding Hou, 1964 \\
A. urdanetensis & (Elmer) Hallier L, 1922 \\
A. yunnanensis & S. C. Huang, 1985 \\
&
\end{tabular}

Knowledge of the main constituents of agarwoods and their major differences between species would be a tremendous help in identifying the different species of agarwood and improving their quality and efficacy of use in traditional medicine. This review will focus on species that are frequently used and reported, including A. malaccensis (synonymous with A. agallocha), A. sinensis, and A. crassna, and will describe the main chemical constituents of agarwoods from different species. The reference content of this article mainly includes literature abstracts and full-text articles from journals, books, reports and electronic searches, including Google Scholar, Elsevier, PubMed, Springer, Web of Science and other related websites. We have selected nearly one hundred articles from different countries, which have been investigated, analyzed and included in this review. This review discusses compounds that have been isolated since 1963.

\section{Sesquiterpenes of agarwoods}

Agarwoods contain various types of sesquiterpenes, which can be divided into several categories depending on their molecular skeletons. Some examples of these sesquiterpenes are agarofurans, agarospiranes, eudesmanes, eremophilanes, guaianes, candinanes, and prezizanes (shown in Scheme 1). Other compounds are also present in small amounts (shown in Scheme 1).

Almost all types of sesquiterpenes can be found in the following four agarwood species: A. sinensis, A. malaccensis, A. crassna, and A. subintegra; however, there are significant differences among the sesquiterpenes of these species, as described in Table 2 .

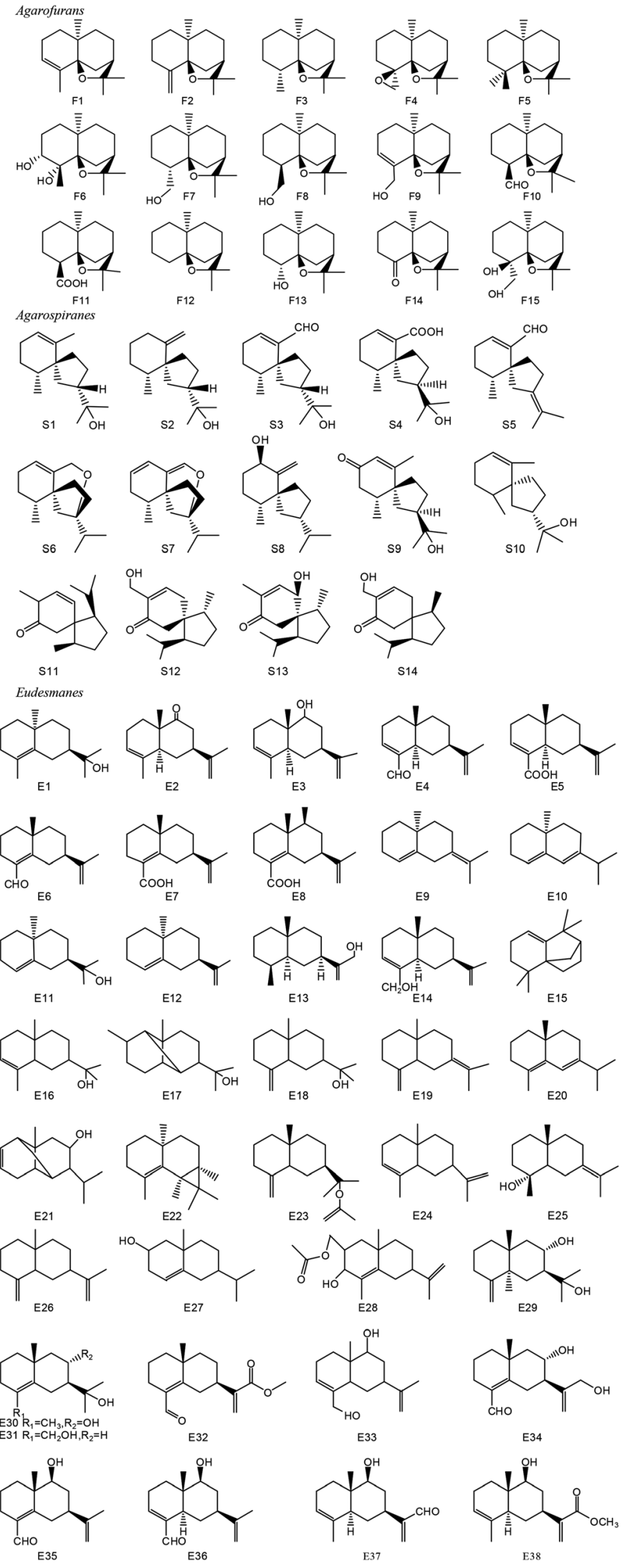

Scheme 1 Different types of sesquiterpenes in agarwood. 
CHO

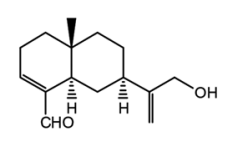

E40

(1)

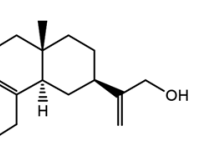

E43

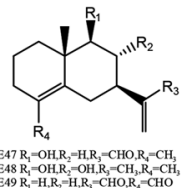

$\mathrm{E} 5 \mathrm{R} \mathrm{R}_{2}=\mathrm{H}, \mathrm{R}_{2}=\mathrm{OH}, \mathrm{R}_{3}=\mathrm{CH}_{3}, \mathrm{R}_{3}=\mathrm{CHO}$
$\mathrm{E} 52 \mathrm{R}_{1}=\mathrm{OH}, \mathrm{CH}_{2}=\mathrm{OH}, \mathrm{H}_{3}=\mathrm{CH}_{3}, \mathrm{CH}_{3}=\mathrm{CH}$

CHO

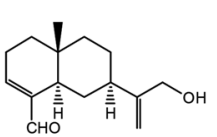

E39

E40

(1)

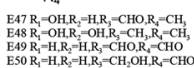

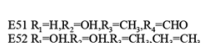

Eremophilanes

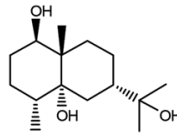

蔩

Guaianes

לT.

(1).

(OHC

CHO $_{1}^{\text {CHO }}$

Tooc

(1)

(1)

(100C

(1)

注

伩

汶

的

争

(1)

(1)

(1)

3

准

ES3 R-OH

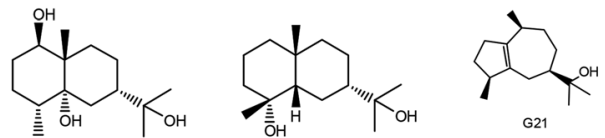

Py

$\underbrace{918}_{\substack{18 \\ 0}}$

(24)

E41

CHO

(1.10H

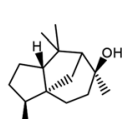

-

制

$x+2$

$\sum_{031}^{626}$

$\overbrace{632}^{627}$

(1)

(1),

$\underbrace{R_{3}}_{R_{4}}$

E53 R $\mathrm{R}=\mathrm{OH}$
$\mathrm{E} 54 \mathrm{R}=\mathrm{H}$

Pot

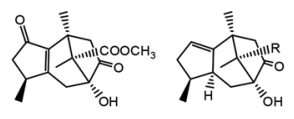

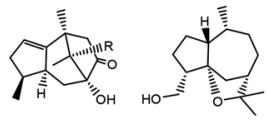

(n)

YOH

(1)
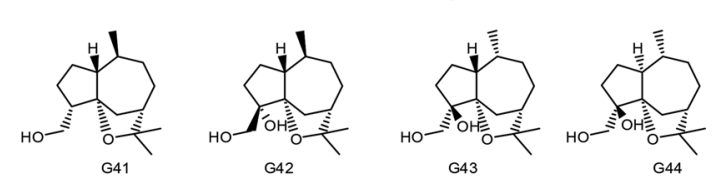

G40

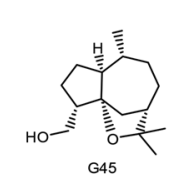

(1)

(1)

(1)

Scheme 1 (contd.)

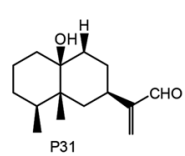



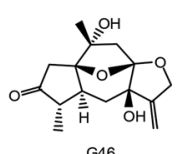

G46

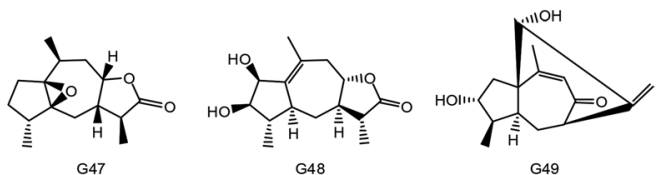

(n)

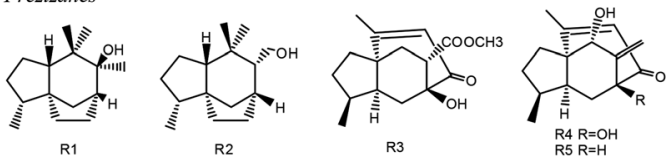

Scheme 1 (contd.) 


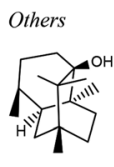

01

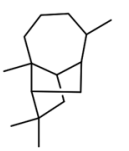

06

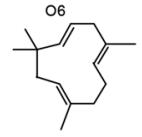

011

016

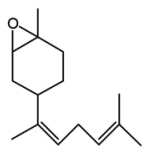

021

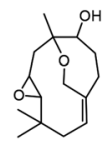

026

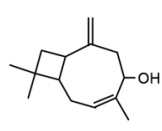

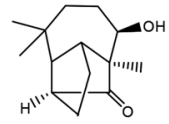

02

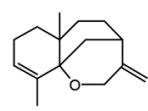

07

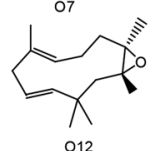

012

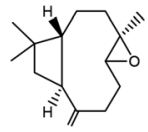

017

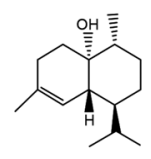

022

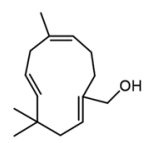

027
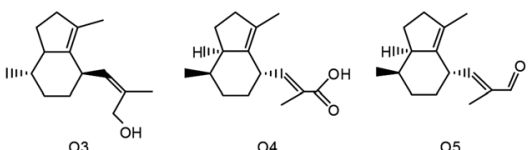

04

05
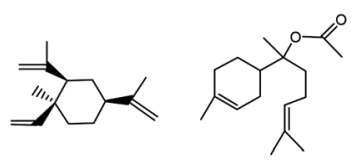

09
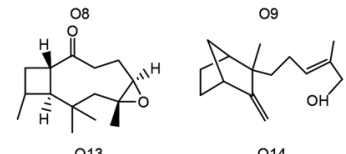

014

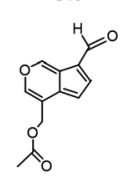

018

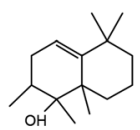

023

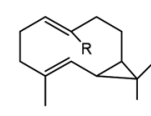

028
Scheme 1 (contd.)

\subsection{Sesquiterpenes in $A$. sinensis}

The sesquiterpenoids of agarwood are mainly derived from agarwood oil. Early publications on agarwood essential oils reflect the fact that the agarwood resin components are separated by solvent extraction, followed by column chromatography for purification and structural analysis using spectroscopy, including NMR. For example, Yang et al. ${ }^{12,21,27}$ and $\mathrm{Xu}$ et al. ${ }^{17}$ isolated sesquiterpenes F1-F2, F7-F9, F11, S1-S3, and G14 from A. sinensis. Yang and coworkers ${ }^{64}$ isolated G19, G23, O12-O13, and $\mathrm{O} 16$ from ethanol and petrol ether extracts of $A$. sinensis and later found two new sesquiterpenes, G27 and O2.

Later articles focused on the use of "combination" techniques to detect and identify compounds. For example, Mei et al. ${ }^{13,42}$ Tian et al. ${ }^{36}$ Deng et al.,${ }^{43}$ Chen et al.,${ }^{2}$ and Miao et al. ${ }^{63}$ detected F3, F5, S10, E1, E9-E11, E15-E22, E24-E28, P1, P3, P6, P8, P13, P15-P16, G13-G14, G16-G18, G21, G26, G30-G34, O1, $\mathrm{O} 5, \mathrm{O} 7, \mathrm{O} 10-\mathrm{O} 11, \mathrm{O} 17, \mathrm{O} 20$, and $\mathrm{O} 19-\mathrm{O} 23$ from essential oils of A. sinensis by GC/MS. Lin $e t$ al. investigated agarwood obtained from fungus-infected Aquilaria at different times by GC-MS and showed the presence of S1, S3, P5, G29-G30, O5, and O18O19. ${ }^{28}$ GC-MS combined with multivariate data analysis was used to construct chemical profiles of natural and artificial agarwoods. The chemical composition of agarwood oil was also studied. Agarwood essential oils are produced by steam distillation or the latest supercritical fluid extraction techniques.
With the development of separation technology, increasing numbers of publications are reporting the separation of sesquiterpenoids from extracts of agarwood resin. The purpose of these studies is to isolate and purify compounds from agarwood, to explore the pharmacological activities of these compounds, and to guide the selection of quality indicators and clinical medication. 'Qi-Nan' is regarded to have the highest quality and is therefore the most expensive agarwood in the market; Yang and coworkers ${ }^{26,37,57}$ performed studies on 'QiNan' originating from A. sinensis and characterized some new sesquiterpenes, including F15, P18, P19, P27, G40-G44, G14, $\mathrm{G} 48, \mathrm{G} 49, \mathrm{~S} 13$, and $\mathrm{S} 12$, from the $\mathrm{Et}_{2} \mathrm{O}$ extract of agarwood. From the ethanolic (EtOH) extract of agarwood induced by artificial holing, Li et al. ${ }^{38,49}$ isolated and identified two new guaiane-type sesquiterpenoids (G46, G47) and eleven eudesmane-type sesquiterpenoids (E34-E44) together with some known sesquiterpenoids, S12, O24, P3, P8, P19, P26, and P27. Kuang and coworkers ${ }^{48}$ were also interested in agarwood induced by artificial holing; they researched the chemical constituents of the $n$-butanol fraction of an EtOH extract and thereby obtained one new sesquiterpene, E33. Huo et al. ${ }^{23} \mathrm{ob}-$ tained nine new sesquiterpenes together with seventeen known ones (E29, E31, E35, E37, E38, E40, E47-E54, F11, P24-P27, P29, $\mathrm{P} 30, \mathrm{O} 28, \mathrm{~S} 3, \mathrm{~S} 9$, and S14) from a 95\% EtOH extract of resinous wood. Zhao et al. $^{32}$ isolated sesquiterpenoids G50-G53, S3, E39, and $\mathrm{E} 45$ from the $95 \% \mathrm{EtOH}$ extract of eaglewood of A. sinensis. Additionally, four sesquiterpenes, E31, P1, P19, and P28, were isolated from the resinous wood of $A$. sinensis in $2018 .^{51}$

\subsection{Sesquiterpenes in A. malaccensis (or A. agallocha)}

A. malaccensis is found in Malaysia, Thailand and India and is currently the most widely distributed species. ${ }^{8}$ The researchers who study this variety are also widely geographically distributed, including Japan, Switzerland, and China. In 1963, Maheshwari and Jain $^{15,20,41}$ isolated and identified F1-F3, F6-F7, F14, and E13 from A. agallocha. From 1992 to 1995, Näf and coworkers found 21 new sesquiterpenes, including agarofurans (F4, F10, and F12F14), agarospiranes (S5-S8), five eudesmanes (E1, E9-E12), and seven eremophilanes (P4-P10 and P12). ${ }^{\mathbf{1 9}, 24,25}$ Ishihara, T. Tsuneya and coworkers found seven eudesmane-type sesquiterpenes (E2E8) and 12 guaiane-type sesquiterpenes (G1-G12, S3, P1, and P3). ${ }^{34,39,55}$ The first agarospirane-type sesquiterpene, $\mathrm{S} 1,^{29,45}$ was found in 1964; later, S9 was obtained from the $70 \%$ ethanol extract of Vietnamese agarwood. ${ }^{35}$ Later, two candinane-type sesquiterpenes, $\mathrm{C} 1$ and $\mathrm{C} 2{ }^{62}$ were found. In 2009, Bhuiyan extracted E26 and G35 (among others) from naturally formed agarwood of A. agallocha as well as from agarwood formed through manual stimulation. ${ }^{45}$ From 1980 to 1983 , Nakanishi ${ }^{30,31}$ discovered three new sesquiterpene alcohols from an agarwood (most likely A. malaccensis), named jinkoh-eremol (P6), jinkohol (R1) and jinkohol II(R2), together with S1 and P8, the major sesquiterpene constituents; their structures have all been established. $\mathrm{Wu}$ and coworkers ${ }^{33,50}$ discovered four new sesquiterpenoids, P27, P31, P32, and E40, together with the four known sesquiterpenoids E55, E45, P3, and S3, all of which were isolated from a $70 \% \mathrm{MeOH}$ extract of $A$. malaccensis agarwood chips. Ma 
Table 2 Sesquiterpenes from different species ${ }^{a, b, c}$

Species

No. Compounds and names

A. $s$

A. $m$

A. $c$

A. $s u$

\section{Agarofurans}

$\alpha$-Agarofuran

$\beta$-Agarofuran

Dihydro- $\beta$-agarofuran

12 and 13

Epoxy- $\beta$-agarofuran

4-Hydroxy-dihydro-agarofuran

3,4-Dihydroxydihydroagarofuran

Baimuxinol

Isobaimuxinol

Dehydrobaimuxinol

$(1 S, 2 S, 6 S, 9 R)-6,10,10$-Trimethyl-11-oxatricyclo[7.2.1.01,6]dodecane-

2-carbaldehyde

Baimuxifuranic acid

12,13 and 17

13

$-$

13

$-$

13 and 21

12 and 13

9

$(1 R, 6 S, 9 R)-6,10,10$-Trimethyl-11-oxatricyclo[7.2.1.01,6]dodecane

21

$(1 R, 2 R, 6 S, 9 R)-6,10,10$-Trimethyl-11-oxatricyclo[7.2.1.01,6]dodecan-2ol

Nor-keto-agarofuran

4-Hydroxyl-baimuxinol

Agarospiranes

S1

$(2 R, 5 R, 10 R)-a, a, 6,10$-tetramethyl-spiro[4,5]dec-6-ene-2-methanol

(agarospirol)

Isoagarospirol

Oxo-agarospirol (baimuxinal)

Baimuxinic acid (Bai Mu Xiang acid)

rel-(5R,10R)-2-Isopropylidene-10-methyl-spiro[4.5]dec-6-ene-6-

carbaldehyde(vetispira-2(11),6-dien-14-al)

rel-(1R,2R)-9-Isopropyl-2-methyl-8-oxatricyclo[7.2.1.01,6]dodec-5-

ene(2,14-epoxy-vetispir-6-ene)

rel-(1R,2R)-9-Isopropyl-2-methyl-8-oxatricyclo[7.2.1.01,6]dodeca-4,6-

dien(2,14-epoxy-vetispira-6(14),7-diene)

rel-(5R,7S,10R)-2-Isopropylidene-10-methyl-6-methylene-spiro[4.5]

decan-7-ol(vetispira-2(11),6(14)-dien-7-ol)

$(4 R, 5 R, 7 R)-1(10)$-Spirovetiven-11-ol-2-one

Hinesol

Acorenone B

4-epi-15-Hydroxyacorenone

4-epi-10-Hydroxyacoronene

15-Hydroxyacorenone

13,22 and 23

-

Eudesmanes

10-epi- $\gamma$-Eudesmol

$(5 S, 7 S, 10 S)$-(-)-Selina-3,11-dien-9-one

$(5 S, 7 S, 9 S, 10 S)-(+)-S e l i n a-3,11-$ dien-9-ol

Selina-3,11-dien-14-al

Selina-3,11-dien-14-oic acid (as methyl ester)

Selina-4,11-dien-14-al

Selina-4,11-dien-14-oic acid (as methyl ester)

9-Hydroxy-selina-4,11-dien-14-oic acid (as methylester)

(S)-4a-Methyl-2-(1-methylethylidene)-1,2,3,4,4a,5,6,7-

octahydronaphthalene

E10

E11

E12

E13

E14

E15

E16

E17

E18
(S)-4a-Methyl-2-(1-methylethyl)-3,4,4a,5,6,7-hexahydronaphthalene 13

$(2 R, 4 a S)-2$-(4a-Methyl-1,2,3,4,4a,5,6,7-octahydronaphthyl)-propan-2- 13

ol(4-nor-epi- $\gamma$-eudesmol)

(2R,4aS)-4a-Methyl-2-(1-methylethenyl)-1,2,3,4,4a,5,6,7-

octahydronaphthalene

Agarol (11(13)-eudesmen-12-ol)

Selina-3,11-dien-14-ol

Isolongifolene

$\alpha$-Eudesmol

$\alpha$-Copaen-11-ol

$\beta$-Eudesmol

-
-
-
36
42
2
13

\section{-}

13, 27 and $28^{*}$

-

$13,17,23,27,28^{*}$

and 32

17 and 27

-

-
-
-

23

2,13 and 36

-

37 and $38^{*}$

37

23

13

-

$-$

-
-
-
-
13
13
13

13 and 42

14 and 15
15 and 18
15 and 16
19
20
20
-
-
-
19

16

16

16

16

$-$

-

-

$-$

$-$

$-$

24

20 and 25

16

16

16 and $29-31 \quad 16$

16

$18 \quad-$

$14,18,33$ and 16

34

$-$

25

25

25

25

35

-

16

-

14, 18 and $25 \quad 16$

16 and $34 \quad 16$

16 and $34 \quad 16$

34 and $39 \quad 16$

39

16 and 39

39

39

24

24

24

24

40 and 41

-

-

-

-

-
-

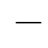

-
16

16

16

16

$-$

16

$-$

$-$
$-$
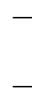

-
16
-
-
- 


\begin{tabular}{|c|c|c|c|c|c|}
\hline \multirow[b]{2}{*}{ No. } & \multirow[b]{2}{*}{ Compounds and names } & \multicolumn{4}{|l|}{ Species } \\
\hline & & A. $s$ & A. $m$ & A. $c$ & A. su \\
\hline E19 & $\gamma$-Selinene & 36,42 and 43 & - & - & - \\
\hline E20 & $\delta$-Selinene & 36 & - & - & - \\
\hline E21 & $\alpha$-Copaene-8-ol & 43 & - & - & - \\
\hline E22 & $\beta$-Maaliene & 36 & - & - & - \\
\hline E23 & $\beta$-Eudesmol acetate & - & - & 16 & 16 \\
\hline E24 & $\alpha$-Selinene & 2 & - & 44 & - \\
\hline E25 & Eudesm-7(11)-en-4a-ol & 2 & - & - & - \\
\hline E26 & Naphthalene, decahydro-7-isopropenyl-4a-methyl-1-methylene- & - & 45- & - & - \\
\hline E27 & $\begin{array}{l}\text { 6-Isopropenyl-4,8a-dimethyl-1,2,3,5,6,7,8,8a-octahydro-naphthalen- } \\
\text { 2-ol }\end{array}$ & 2 & - & - & - \\
\hline E28 & $\begin{array}{l}\text { Acetic acid, 3-hydroxy-6-isopropenyl-4,8 } a \text {-dimethyl-1,2,3,5,6,7,8,8a- } \\
\text { octahydronaphthalen-2-yl ester }\end{array}$ & 2 & - & - & - \\
\hline E29 & 5-Desoxylongilobol & 23 and 46 & - & 46 & - \\
\hline E30 & Eudesma-4-en-8,11-diol & - & - & 47 & - \\
\hline E31 & Eudesma-4-en-11,15-diol & 23 & - & 47 & - \\
\hline E32 & Methyl-15-oxo-eudesmane-4,11(13)-dien-12-oate & - & - & 47 & - \\
\hline E33 & Selina-3,11-dien-9,15-diol & $48^{*}$ & - & - & - \\
\hline E34 & $(7 S, 8 R, 10 S)-(+)-8,12-D i h y d r o x y-s e l i n a-4,11-d i e n-14-a l$ & $49^{*}$ & - & - & - \\
\hline E35 & $(7 S, 9 S, 10 S)-(+)-9-H y d r o x y-s e l i n a-4,11-d i e n-14-a l$ & 23 and $49^{*}$ & - & - & - \\
\hline E36 & $(5 S, 7 S, 9 S, 10 S)-(-)-9-H y d r o x y-s e l i n a-3,11-d i e n-14-a l$ & $49^{*}$ & - & - & - \\
\hline E37 & $(5 S, 7 S, 9 S, 10 S)-(+)-9-H y d r o x y-s e l i n a-3,11-d i e n-12-a l$ & 23 and $49^{*}$ & - & - & - \\
\hline E38 & $(5 S, 7 S, 9 S, 10 S)-(+)-9-H y d r o x y-e u d e s m a-3,11(13)-d i e n-12-m e t h y l e s t e r$ & 23 and $49^{*}$ & - & - & - \\
\hline E39 & Selina-3,11-diene-12,15-dial (=12,15-dioxo- $\alpha$-selinen $)$ & 32 and $49^{*}$ & - & - & - \\
\hline $\mathrm{E} 40$ & $\begin{array}{l}(4 \alpha \beta, 7 \beta, 8 \alpha \beta)-3,4,4 \alpha, 5,6,7,8,8 \alpha \text {-Octahydro-7-[1-(hydroxymethyl) } \\
\text { ethenyl]-4 } 4 \alpha \text {-methylnaphthalene-1-carboxaldehyde }\end{array}$ & 23 and $49^{*}$ & 50 & - & - \\
\hline E41 & Eudesmane-1 $\beta, 5 \alpha, 11$-triol & $49^{*}$ & - & - & - \\
\hline $\mathrm{E} 42$ & $(-)-7 \beta H$-Eudesmane- $4 \alpha, 11$-diol & $49^{*}$ & - & - & - \\
\hline $\mathrm{E} 43$ & ent-4(15)-Eudesmen-11-ol-1-one & $49^{*}$ & - & - & - \\
\hline E44 & 15-Hydroxyl-12-oxo- $\alpha$-selinen & $49^{*}$ & - & - & - \\
\hline $\mathrm{E} 45$ & Selina-4,11-diene-12,15-dial & 32 & 50 & - & - \\
\hline E46 & (+)-Eudesma-4(14),11(13)-dien-8 $\alpha, 9 \beta$-diol & 23 & - & - & - \\
\hline E47 & (+)-9ß-Hydroxyeudesma-4,11(13)-dien-12-al & 23 & - & - & - \\
\hline $\mathrm{E} 48$ & (+)-Eudesma-4,11(13)-dien-8 $\alpha, 9 \beta$-diol & 23 & - & - & - \\
\hline E49 & 12,15-Dioxo-selina-4,11-dine & 23 & - & - & - \\
\hline E50 & 12-Hydroxy-4(5),11(13)-eudesmadien-15-al & 23 & - & - & - \\
\hline E51 & (+)-8 $\alpha$-Hydroxyeudesma-3,11(13)-dien-14-al & 23 & - & - & - \\
\hline E52 & (+)-Eudesma-3,11(13)-dien- $8 \alpha, 9 \beta$-diol & 23 & - & - & - \\
\hline E53 & $(4 R, 5 R, 7 S, 9 S, 10 S)-(-)-E u d e s m a-11(13)-e n-4,9-d i o l$ & 23 & - & - & - \\
\hline E54 & Selin-11-en- $4 \alpha$-ol & 23 & - & - & - \\
\hline E55 & $\begin{array}{l}\text { Eudesm-4-ene-11,15-diol } \\
\text { Eremophilanes }\end{array}$ & 23 & 50 & - & - \\
\hline $\mathrm{P} 1$ & $(+)-(4 S, 5 R)$-Dihydrokaranone & 13 and 51 & 18 and 34 & - & - \\
\hline $\mathrm{P} 2$ & $(+)-(4 S, 5 R)$-karanone & - & 18 & 16 & 16 \\
\hline P3 & Eremophila-9,11-dien-8-one (neopetasane) & $2,13,26,38^{*}$ and 51 & 16,33 and 39 & 16 & 16 \\
\hline $\mathrm{P} 4$ & 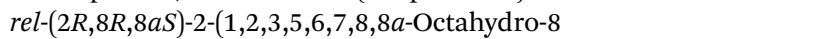 & - & 25 & - & - \\
\hline P5 & 8,12-Epoxy-eremophila-9,11(13)-diene & $28^{*}$ & 25 & - & - \\
\hline P6 & $(-)-(4 R, 5 S, 7 R)$-Jinkoh-eremol & 13 & 25,30 and 39 & 16 & 16 \\
\hline P7 & Dehydro-jinkoh-eremol & - & 16,25 and 39 & 16 & 16 \\
\hline P8 & $(+)-(4 R, 5 S, 7 R)$-Kusunol (=valerianol) & $13,38^{*}$ and 52 & 14,25 and 30 & 16 & 16 \\
\hline P9 & $\begin{array}{l}\text { rel-( } 2 R, 8 S, 8 a S)-2-(1,2,6,7,8,8 a \text {-Hexahydro-8,8a-dimethyl-2-naphthyl)- } \\
\text { propan-2-ol(valenca-1(10),8-dien-11-ol) }\end{array}$ & - & 25 & - & - \\
\hline $\mathrm{P} 10$ & Valenc- or eremophil-9-en-12-al(tentative) & - & 25 & - & - \\
\hline P11 & Calarene & - & 53 & - & - \\
\hline $\mathrm{P} 12$ & 2,t-3-Dimethyl-r-2-(3-methyl-2-butenyl)-1-cyclohexanone & - & 19 & - & - \\
\hline $\mathrm{P} 13$ & Valencene & 42 and 43 & - & 54 & - \\
\hline P14 & Aristolone & - & - & 54 & - \\
\hline P15 & Aristolene & 42 & - & - & - \\
\hline $\mathrm{P} 16$ & Nootkatone & 42 & - & - & - \\
\hline $\mathrm{P} 17$ & Calarene & - & - & 54 & - \\
\hline P18 & $7 b$-H-9(10)-ene-11,12-epoxy-8-oxoeremophilane & 26 & - & - & - \\
\hline P19 & $7 \alpha-H$-9(10)-ene-11,12-epoxy-8-oxoeremophilane & $26,38^{*}, 46$ and 51 & - & 46 & - \\
\hline
\end{tabular}


Table 2 (Contd.)

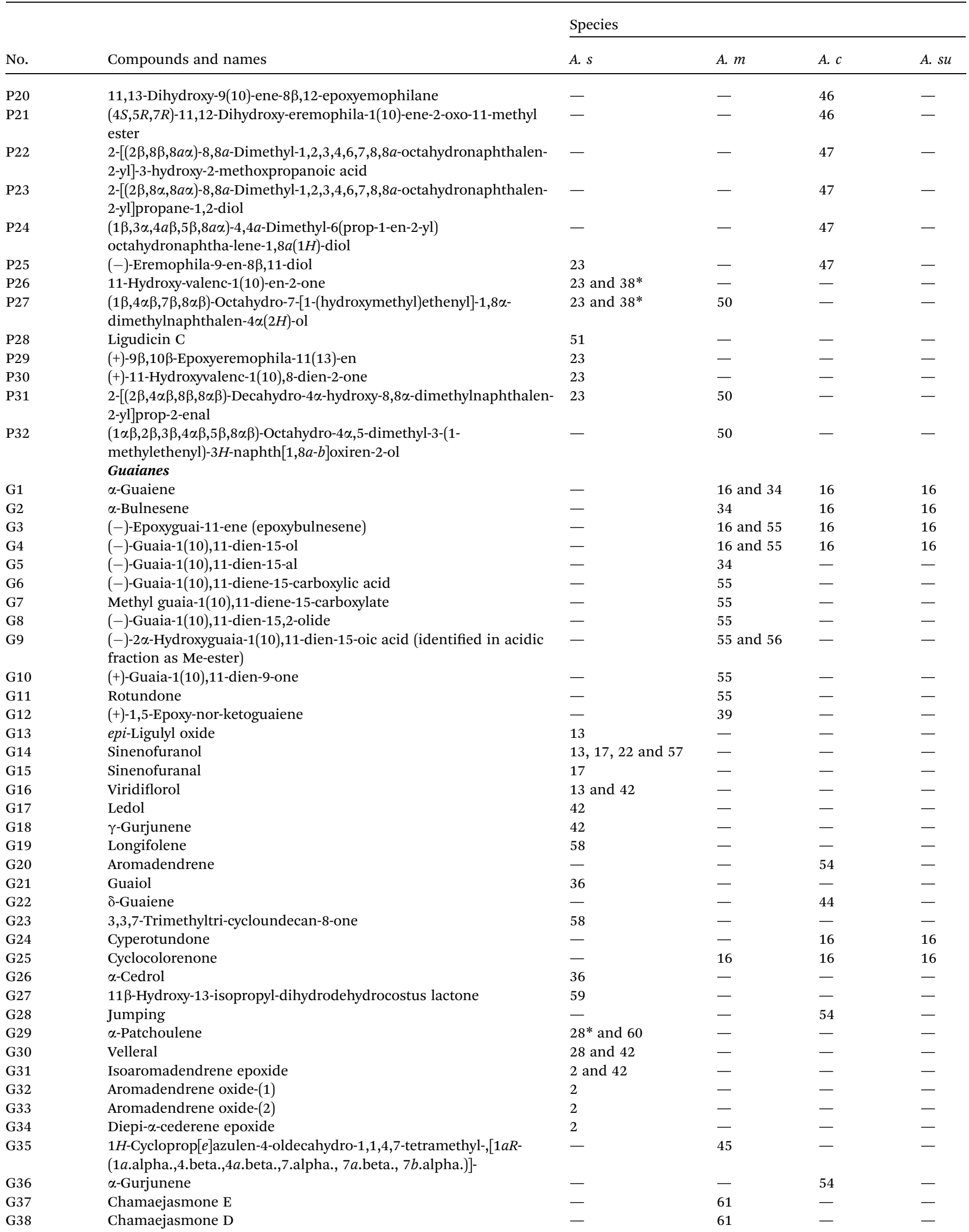




\begin{tabular}{|c|c|c|c|c|c|}
\hline \multirow[b]{2}{*}{ No. } & \multirow[b]{2}{*}{ Compounds and names } & \multicolumn{4}{|l|}{ Species } \\
\hline & & A. $s$ & A. $m$ & A. $c$ & A. su \\
\hline G39 & Auranticanol A & - & 61 & - & - \\
\hline G40 & Qinanol A & 57 & - & - & - \\
\hline G41 & Qinanol B & 57 & - & - & - \\
\hline G42 & Qinanol C & 57 & - & - & - \\
\hline G43 & Qinanol D & 57 & - & - & - \\
\hline G44 & Qinanol E & 57 & - & - & - \\
\hline G45 & Sinenofuranol & 57 & - & - & - \\
\hline G46 & 3-Oxo-7-hydroxylholosericin A & $38^{*}$ & - & - & - \\
\hline G47 & 1,5;8,12-Diepoxyguaia-12-one & $38^{*}$ & - & - & - \\
\hline G48 & Qinanlactone & 37 & - & - & - \\
\hline G49 & Qinan-guaiane-one & 37 & - & - & - \\
\hline G50 & 7H-Guaia-1(10)-en-12,8-olide & 32 & - & - & - \\
\hline G51 & 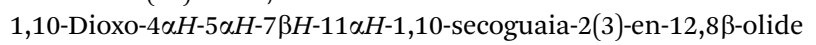 & 32 & - & - & - \\
\hline G52 & $1 \alpha$-Hydroxy- $4 \beta H-5 \beta H-7 \beta H-11 \alpha H-8,9$-secoguaia-9(10)-en-8,12-olide & 32 & - & - & - \\
\hline G53 & $\begin{array}{l}1 \alpha \text {-Hydroxy- } 4 \alpha, 10 \alpha \text {-dimethyl-5 } \beta \text { H-octahydro-azulen-8-one } \\
\text { Candinanes }\end{array}$ & 32 & - & - & - \\
\hline $\mathrm{C} 1$ & $8 \beta H$-Dihydrogmelofuran & - & 62 & - & - \\
\hline $\mathrm{C} 2$ & Gmelofuran & - & 62 & - & - \\
\hline C3 & $\begin{array}{l}(7 \beta, 8 \beta, 9 \beta)-8,9-E p o x y c a l a m e n e n-10-o n e \\
\text { Prezizanes }\end{array}$ & - & - & 46 & - \\
\hline $\mathrm{R} 1$ & Jinkohol & - & 30 and 31 & - & - \\
\hline $\mathrm{R} 2$ & Jinkohol II & - & 30 & - & - \\
\hline R3 & Daphnauranol B & - & 61 & - & - \\
\hline $\mathrm{R} 4$ & Daphnauranol C & - & 61 & - & - \\
\hline R5 & $\begin{array}{l}\text { Daphnauranol D } \\
\text { Others }\end{array}$ & - & 61 & - & - \\
\hline O1 & Patchoulialcohol & 43 & - & - & - \\
\hline $\mathrm{O} 2$ & $(+)-8 \beta$-Hydroxy-longicamphenylone & 59 & - & - & - \\
\hline $\mathrm{O} 3$ & Valerenol & - & - & 54 & - \\
\hline $\mathrm{O} 4$ & Valerenic acid & 42 & - & 54 & - \\
\hline O5 & Valerenal & $28^{*}$ & - & 54 & - \\
\hline O6 & Dihydro-neoclovene & - & - & 54 & - \\
\hline O7 & 2,6-Dimethyl-10-methylene-12-oxatricyclo[7.3.1.0(1,6)]tridec-2-ene & 2 & - & - & - \\
\hline O8 & $\beta$-Elemene & - & - & 16 & 16 \\
\hline O9 & $\alpha$-Bisabolol acetate & - & - & - & 16 \\
\hline O10 & $\beta$-Caryophyllene & 43 & - & - & - \\
\hline O11 & $\alpha$-Humulene & 43 & - & - & - \\
\hline O12 & Humulene diepoxide A & 58 & - & - & - \\
\hline O13 & Kobusone & 58 & - & - & - \\
\hline O14 & Santalol & 36,42 and 58 & - & - & - \\
\hline O15 & $(E)$-Nerolidol & - & 16 and 18 & 16 & 16 \\
\hline O16 & Caryophyllenol-II & 58 & - & - & - \\
\hline O17 & Caryophylleneoxide & 2,42 and 43 & 45 & - & - \\
\hline O18 & Baldrinal & $28 *$ & - & - & - \\
\hline O19 & $\alpha$-Muurolene & $28^{*}$ and 63 & - & - & - \\
\hline $\mathrm{O} 20$ & Elemol & 2 and 13 & - & - & - \\
\hline $\mathrm{O} 21$ & cis-Z- $\alpha$-Bisabolene epoxide & 2 & - & - & - \\
\hline $\mathrm{O} 22$ & Cubenol & 2 & - & - & - \\
\hline $\mathrm{O} 23$ & 1,2,5,5,8a-Pentamethyl-1,2,3,5,6,7,8,8a-octahydronaphthalen-1-ol & 2 & - & - & - \\
\hline $\mathrm{O} 24$ & 1,5,9-Trimethyl-1,5,9-cyclododecatriene & $38^{*}$ & - & - & - \\
\hline $\mathrm{O} 25$ & Aquilanol A & - & 61 & - & - \\
\hline $\mathrm{O} 26$ & Aquilanol B & - & 61 & - & - \\
\hline $\mathrm{O} 27$ & 12-Hydroxyhumula-2Z,6E,9E-triene & - & 61 & - & - \\
\hline $\mathrm{O} 28$ & 14-Hydroxy- $\alpha$-humulene & 23 & - & - & - \\
\hline
\end{tabular}

${ }^{a}$ A. $s, A . m, A$. c , and A. su indicate A. sinensis, A. malaccensis, A. crassna, and A. subintegra, respectively. ${ }^{b}$ The reference was not found. ${ }^{c}$ "*” indicates that the agarwood in this article was artificial agarwood. 
2-(2-Phenylethyl)chromones

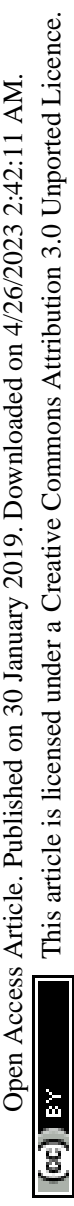
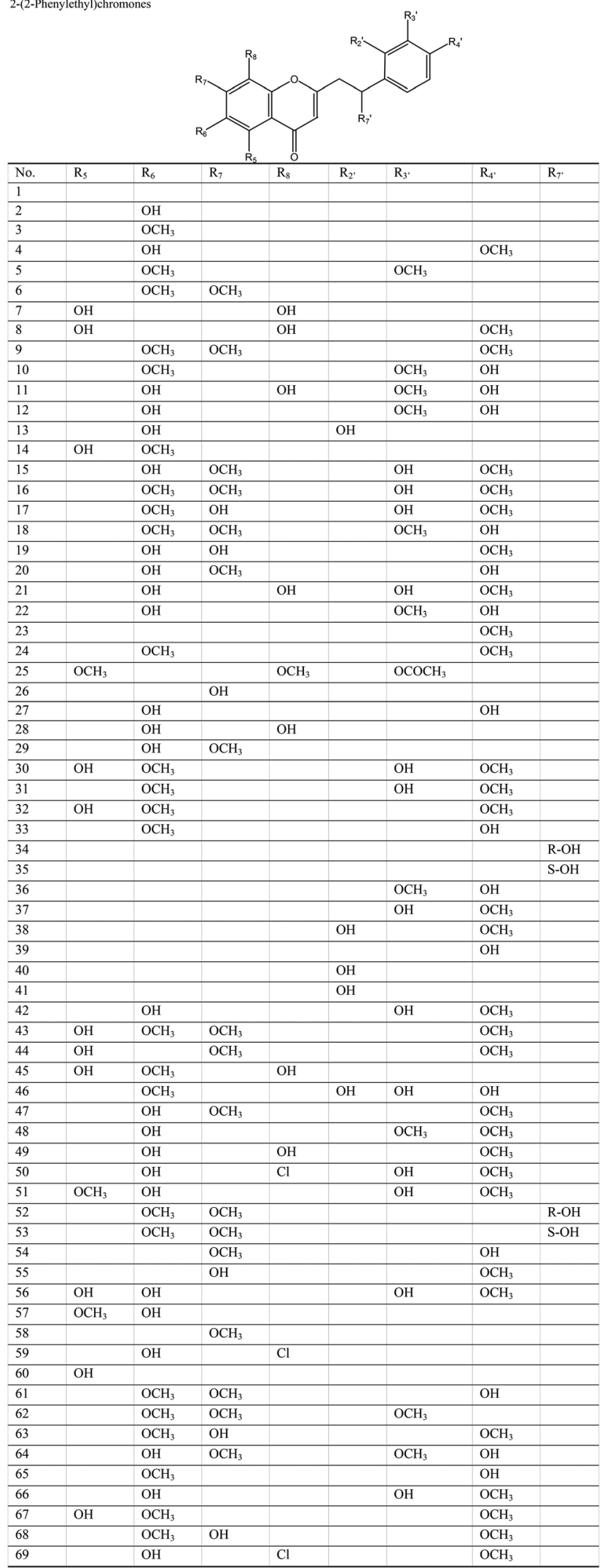

Scheme 2 Different types of chromone derivatives in agarwood
5, 6, 7, 8-Tetrahydro-2-(2-phenylethyl)chromones

\begin{tabular}{|c|c|c|c|c|c|c|c|c|}
\hline No. & $\mathbf{R}_{5}$ & $\mathbf{R}_{6}$ & $\mathbf{R}_{7}$ & $\mathrm{R}_{8}$ & $\mathbf{R}_{2}$, & $\mathbf{R}_{3}$, & $\mathbf{R}_{4}$ & $\mathbf{R}_{7}$ \\
\hline 70 & & $\beta-\mathrm{OH}$ & $\beta-\mathrm{OH}$ & & & & & \\
\hline 71 & $\alpha-\mathrm{OH}$ & $\alpha-\mathrm{OH}$ & $\alpha-\mathrm{OH}$ & $\beta$-Cl & & & & \\
\hline 72 & $\alpha-\mathrm{OH}$ & $\alpha-\mathrm{OH}$ & $\alpha-\mathrm{OH}$ & $\beta-\mathrm{Cl}$ & & $\mathrm{OH}$ & $\mathrm{OCH}_{3}$ & \\
\hline 73 & $\alpha-\mathrm{OH}$ & $\alpha-\mathrm{OH}$ & $\beta-\mathrm{OH}$ & $\alpha-\mathrm{OH}$ & & $\mathrm{OH}$ & $\mathrm{OCH}_{3}$ & \\
\hline 74 & $\alpha-\mathrm{OH}$ & $\beta-\mathrm{OH}$ & $\alpha-\mathrm{OH}$ & $\beta-\mathrm{OH}$ & & $\mathrm{OH}$ & $\mathrm{OCH}_{3}$ & \\
\hline 75 & $\alpha-\mathrm{OH}$ & $\alpha-\mathrm{OH}$ & $\beta-\mathrm{OH}$ & & & $\mathrm{OH}$ & $\mathrm{OCH}_{3}$ & \\
\hline 76 & $\alpha-\mathrm{OH}$ & $\beta-\mathrm{OH}$ & $\beta-\mathrm{OH}$ & & & $\mathrm{OH}$ & $\mathrm{OCH}_{3}$ & \\
\hline 77 & $\alpha-\mathrm{OH}$ & $\beta-\mathrm{OH}$ & $\beta-\mathrm{OH}$ & $\alpha-\mathrm{OH}$ & & & & \\
\hline 78 & $\alpha-\mathrm{OH}$ & $\beta-\mathrm{OH}$ & $\alpha-\mathrm{OH}$ & $\beta-\mathrm{OH}$ & & & & \\
\hline 79 & $\beta-\mathrm{OH}$ & $\beta-\mathrm{OH}$ & $\alpha-\mathrm{OH}$ & $\beta$-OH & & & & \\
\hline 80 & $\alpha-\mathrm{OH}$ & $\beta-\mathrm{OH}$ & $\alpha-\mathrm{OH}$ & $\beta$-OH & $\mathrm{OH}$ & & & \\
\hline 81 & $\alpha-\mathrm{OH}$ & $\beta-\mathrm{OH}$ & $\alpha-\mathrm{OH}$ & $\beta-\mathrm{OH}$ & & & & $\alpha-\mathrm{OH}$ \\
\hline 82 & $\alpha-\mathrm{OH}$ & $\beta-\mathrm{OH}$ & $\beta-\mathrm{OH}$ & $\alpha-\mathrm{OH}$ & $\mathrm{OH}$ & & & \\
\hline 83 & $\alpha-\mathrm{OH}$ & $\beta-\mathrm{OH}$ & $\alpha-\mathrm{OH}$ & $\beta-\mathrm{OH}$ & & & $\mathrm{OCH}_{3}$ & \\
\hline 84 & $\alpha-\mathrm{OCOCH}_{3}$ & $\beta-\mathrm{OCOCH}_{3}$ & $\beta-\mathrm{OCOCH}_{3}$ & $\alpha-\mathrm{OCOCH}_{3}$ & & & $\mathrm{OCH}_{3}$ & \\
\hline 85 & $\alpha-\mathrm{OCOCH}_{3}$ & $\beta-\mathrm{OCOCH}_{3}$ & $\alpha-\mathrm{OCOCH}_{3}$ & & $\mathrm{OCOCH}_{3}$ & & & \\
\hline 86 & $\alpha-\mathrm{OCH}_{3}$ & $\alpha-\mathrm{OH}$ & $\beta-\mathrm{OH}$ & $\alpha-\mathrm{OH}$ & & & $\mathrm{OCH}_{3}$ & \\
\hline 87 & $\beta-\mathrm{CH}_{3}$ & $\beta-\mathrm{OH}$ & $\alpha-\mathrm{OH}$ & $\beta-\mathrm{OH}$ & & & & \\
\hline 88 & $\alpha-\mathrm{OCH}_{3}$ & $\alpha-\mathrm{OH}$ & $\beta-\mathrm{OH}$ & $\alpha-\mathrm{OH}$ & & $\mathrm{OH}$ & $\mathrm{OCH}_{3}$ & \\
\hline 89 & $\alpha-\mathrm{OCH}_{3}$ & $\alpha-\mathrm{OH}$ & $\beta-\mathrm{OH}$ & $\alpha-\mathrm{Cl}$ & & & $\mathrm{OCH}_{3}$ & \\
\hline 90 & $\alpha-\mathrm{OH}$ & $\alpha-\mathrm{OH}$ & $\beta-\mathrm{OH}$ & $\alpha-\mathrm{OCH}_{3}$ & & & $\mathrm{OCH}_{3}$ & \\
\hline 91 & $\alpha-\mathrm{OCH}_{3}$ & $\beta-\mathrm{OH}$ & $\alpha-\mathrm{OH}$ & $\beta-\mathrm{OH}$ & & & $\mathrm{OCH}_{3}$ & \\
\hline 92 & $\beta-\mathrm{OCH}_{3}$ & $\alpha-\mathrm{OH}$ & $\beta-\mathrm{OH}$ & $\alpha-\mathrm{OH}$ & & & $\mathrm{OCH}_{3}$ & \\
\hline 93 & $\alpha-\mathrm{OCH}_{3}$ & $\beta-\mathrm{OH}$ & $\alpha-\mathrm{OH}$ & $\beta-\mathrm{OH}$ & & $\mathrm{OH}$ & $\mathrm{OCH}_{3}$ & \\
\hline 94 & $\alpha-\mathrm{OCH}_{3}$ & $\beta-\mathrm{OH}$ & $\alpha-\mathrm{OH}$ & $\beta-\mathrm{Cl}$ & & & $\mathrm{OCH}_{3}$ & \\
\hline 95 & $\alpha-\mathrm{OCH}_{3}$ & $\beta$-OH & $\alpha-\mathrm{OH}$ & $\beta-\mathrm{Cl}$ & & $\mathrm{OH}$ & $\mathrm{OCH}_{3}$ & \\
\hline 96 & $\alpha-\mathrm{OH}$ & $\beta-\mathrm{OH}$ & $\beta-\mathrm{OH}$ & $\alpha-\mathrm{OCH}_{3}$ & & & $\mathrm{OCH}_{3}$ & \\
\hline 97 & $\beta-\mathrm{OH}$ & $\alpha-\mathrm{OH}$ & $\alpha-\mathrm{OH}$ & $\beta-\mathrm{Cl}$ & & & $\mathrm{OCH}_{3}$ & \\
\hline 98 & $\alpha-\mathrm{OH}$ & $\alpha-\mathrm{OH}$ & $\alpha-\mathrm{OH}$ & $\beta$-OH & & $\mathrm{OH}$ & $\mathrm{OCH}_{3}$ & \\
\hline 99 & $\alpha-\mathrm{OH}$ & $\alpha-\mathrm{OH}$ & $\alpha-\mathrm{OH}$ & $\beta-\mathrm{OH}$ & & & & \\
\hline 100 & $\alpha-\mathrm{OH}$ & $\alpha-\mathrm{OH}$ & $\alpha-\mathrm{OH}$ & $\beta-\mathrm{OH}$ & & & $\mathrm{OCH}_{3}$ & \\
\hline 101 & $\alpha-\mathrm{OH}$ & $\beta-\mathrm{OH}$ & $\beta-\mathrm{OH}$ & $\alpha-\mathrm{OH}$ & & $\mathrm{OH}$ & $\mathrm{OCH}_{3}$ & \\
\hline 102 & $\beta-\mathrm{OH}$ & $\beta$-OH & $\alpha-\mathrm{OH}$ & $\beta-\mathrm{Cl}$ & & & $\mathrm{OCH}_{3}$ & \\
\hline 103 & $\alpha-\mathrm{OH}$ & $\alpha-\mathrm{OH}$ & $\alpha-\mathrm{OH}$ & $\alpha-\mathrm{Cl}$ & & & & \\
\hline 104 & $\beta-\mathrm{OH}$ & $\beta-\mathrm{OH}$ & $\beta-\mathrm{OH}$ & $\beta-\mathrm{Cl}$ & & & $\mathrm{OCH}_{3}$ & \\
\hline 105 & $\beta-\mathrm{OH}$ & $\alpha-\mathrm{OH}$ & $\alpha-\mathrm{OH}$ & & & $\mathrm{OCH}_{3}$ & $\mathrm{OH}$ & \\
\hline 106 & $\alpha-\mathrm{OH}$ & $\beta$-OH & $\beta-\mathrm{OH}$ & $\alpha-\mathrm{OH}$ & & & $\mathrm{OH}$ & \\
\hline 107 & $\alpha-\mathrm{OH}$ & $\alpha-\mathrm{OH}$ & $\beta-\mathrm{OH}$ & $\alpha-\mathrm{Cl}$ & & $\mathrm{OH}$ & $\mathrm{OCH}_{3}$ & \\
\hline 108 & $\beta-\mathrm{OH}$ & $\alpha-\mathrm{OH}$ & $\beta-\mathrm{OH}$ & & & & & \\
\hline 109 & $\beta-\mathrm{OH}$ & $\alpha-\mathrm{OH}$ & $\beta-\mathrm{OH}$ & & & & $\mathrm{OCH}_{3}$ & \\
\hline
\end{tabular}

Diepoxy-tetrahydro-2-(2-phenylethyl)chromones<smiles>[R9]c1ccc(CCc2cc(=O)c3c(o2)C2CO[C@H]2[C@H]2O[C@@H]32)cc1Br</smiles>

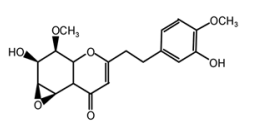

113

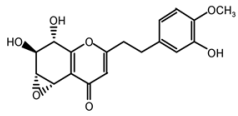

117<smiles>C[C@H]1[C@@H](CO)O[C@@H](Oc2ccc(CCCC3CC(=O)c4ccccc4O3)cc2O)[C@H](O)[C@@H]1O</smiles>

121

Scheme 2 (contd.)

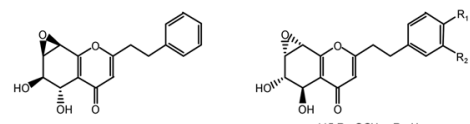

$115 \mathrm{R}_{2}=\mathrm{OCH} \mathrm{H}_{2}, \mathrm{R}_{2}=\mathrm{H}$
$116 \mathrm{R}_{1}=\mathrm{OCH} \mathrm{H}_{3}, \mathrm{R}_{2}=\mathrm{OH}$

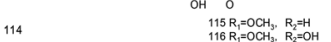<smiles>Cc1ccc(CCC2CC(=O)C3C(=O)[C@@H](C)[C@@H](O)[C@H]3O2)cc1I</smiles>

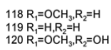

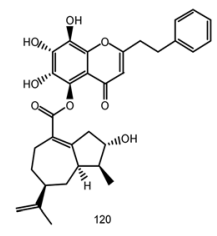



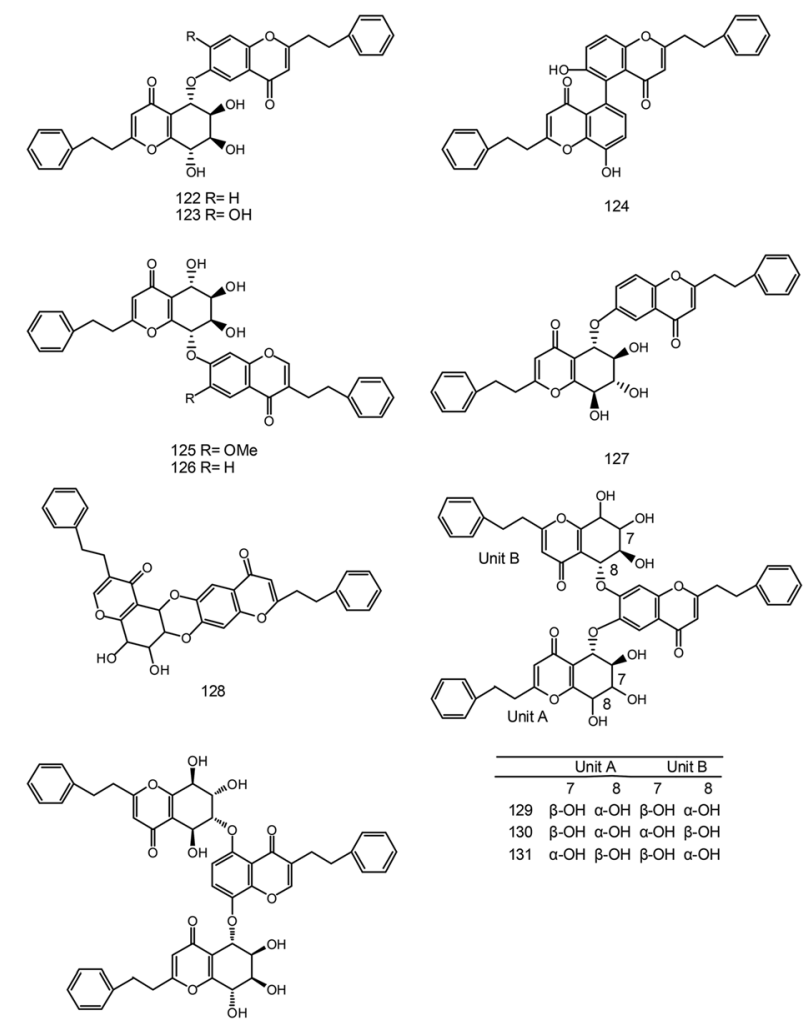

$131 \mathrm{\alpha}-\mathrm{OH} \beta-\mathrm{OH} \beta-\mathrm{OH} \alpha-\mathrm{OH}$

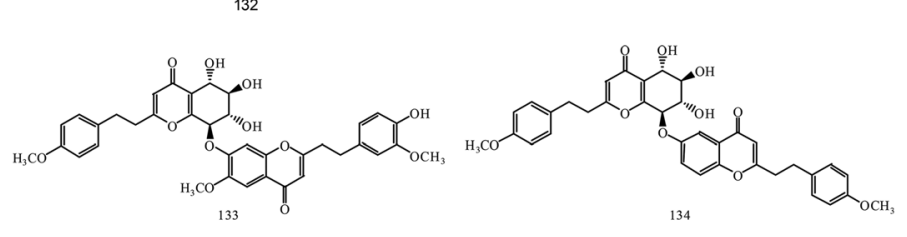

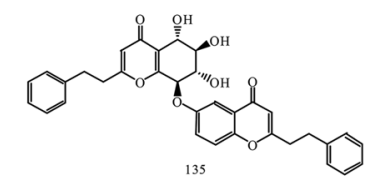

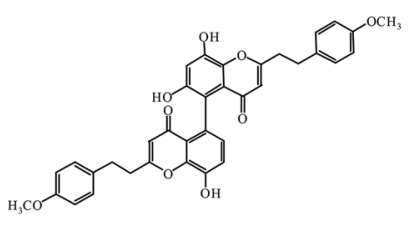

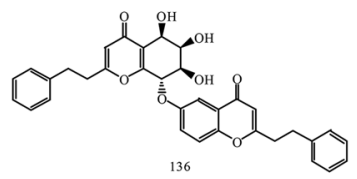

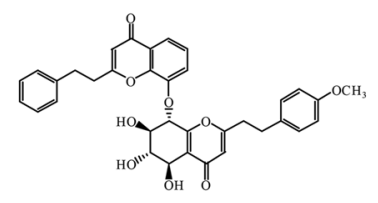

138

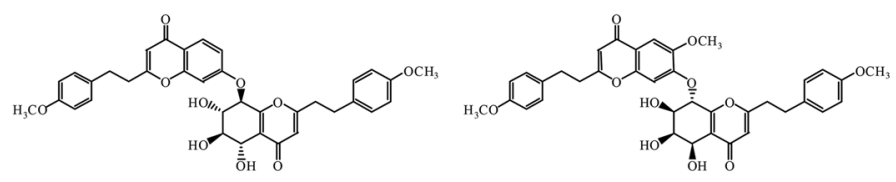

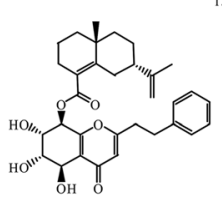

141

Scheme 2 (contd.)

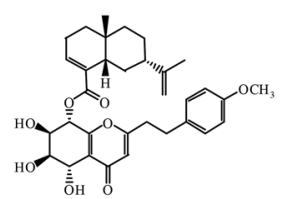

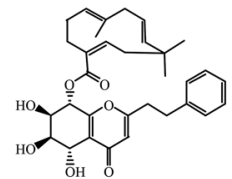

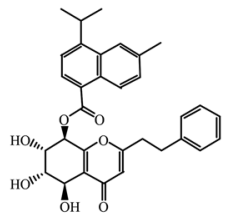

145

146

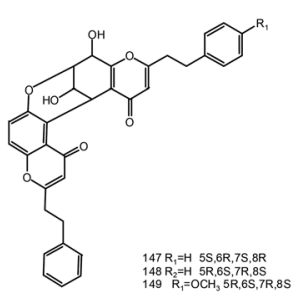

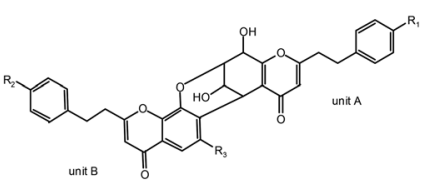

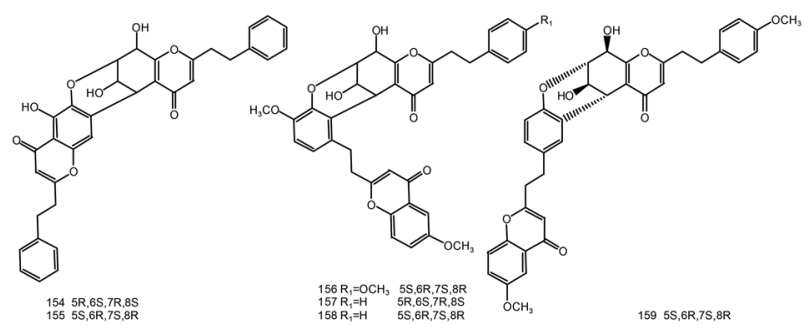

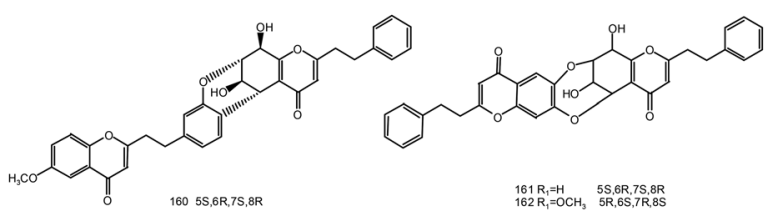

Scheme 2 (contd.)

et al. ${ }^{61}$ reported sesquiterpenoids O25-O27, G37-G39, and R3-R5 from the ether extract of agarwood of $A$. malaccensis.

\subsection{Sesquiterpenes in A. crassna and A. subintegra}

There are currently few studies on A. crassna and A. subintegra. ${ }^{8}$ In 2001, Pripdeevech and coworkers analyzed the diversity of compounds in A. malaccensis, A. crassna, and A. subintegra by GC-MS and GC-O. The study suggested that these species share sesquiterpenes F3, F15, S3, S11, E2, E3, E6, E18, P3, P7, G1, G3, $\mathrm{G} 4, \mathrm{G} 25$, O14, and $015 .{ }^{16} \mathrm{E} 24$ and G22 were found in the supercritical carbon dioxide extraction of A. crassna $^{44}$ Pornpunyapat, Chetpattananondh and Tongurai, assessed the extraction conditions of essential oils obtained from A. crassna and detected P13-P14, P17, G20, G28, G36, and O3-O6. ${ }^{54}$

Wang et al. ${ }^{46}$ isolated and identified the sesquiterpenoids C3, P19, E2 and E29 from the ethyl acetate (EtOAc) extract of agarwood originating from A. crassna. The following year, Kang and Dai et al. ${ }^{47}$ separated the sesquiterpenes P22-P25 and E30-E32 from a $95 \% \mathrm{EtOH}$ extract of agarwood from A. crassna.

\section{Chromone derivatives in agarwoods}

Chromone derivatives are other major constituents of agarwoods. They have been obtained from only a few plant species, including Eremophila georgei, Bothriochloa ischaemum 
Table 3 Chromones from different species ${ }^{a, b, c}$

Species

\section{A. $s$}

A. $m$

A. $c$

\section{2-(2-Phenylethyl)chromones}

2-(2-Phenylethyl)chromone (flindersiachromone)

6-Hydroxy-2-(2-phenylethyl)chromone $\left(\mathrm{AH}_{3}\right)$

6-Methoxy-2-(2-phenylethyl)chromone $\left(\mathrm{AH}_{4}\right)$

6-Hydroxy-2-[2-(4-methoxyphenyl)ethyl]chromone

6-Methoxy-2-[2-(3-methoxyphenyl)ethyl]chromone $\left(\mathrm{AH}_{5}\right)$

6,7-Dimethoxy-2-(2-phenylethyl)chromone $\left(\mathrm{AH}_{6}\right)$

5,8-Dihydroxy-2-(2-phenylethyl)chromone $\left(\mathrm{AH}_{7}\right)$

5,8-Dihydroxy-2-[2-(4-methoxyphenyl)ethyl]chromone

6,7-Dimethoxy-2-[2-(4'-methoxyphenyl)ethyl]chromone $\left(\mathrm{AH}_{8}\right)$

6-Methoxy-2-[2-(3-methoxy-4-hydroxyphenyl)ethyl]chromone

6,8-Dihydroxy-2-[2-(3-methoxy-4-hydroxyphenyl)ethyl]chromone

6-Hydroxy-2-[2-(3-methoxy-4-hydroxyphenyl)ethyl]chromone

6-Hydroxy-2-(2'-hydroxy-2-phenylethyl)chromone

5-Hydroxy-6-methoxy-2-(2-phenylethyl)chromone

6-Hydroxy-7-methoxy-2-[2-(3'-hydroxy-4'-methoxyphenyl)ethyl]chromone

6,7-Dimethoxy-2-[2-(3'-hydroxy-4'-methoxyphenyl)ethyl]chromone

7-Hydroxy-6-methoxy-2-[2-(3'-hydroxy-4'-methoxy-phenyl)ethyl]chromone 6,7-Dimethoxy-2-[2-(4'-hydroxy-3'-methoxyphenyl)ethyl]chromone

6,7-Dihydroxy-2-[2-(4'-methoxyphenyl)ethyl]chromone

6-Hydroxy-7-methoxy-2-[2-(4'-hydroxyphenyl)ethyl]chromone

6,8-Dihydroxy-2-[2-(3'-hydroxy-4'-methoxyphenyl)ethyl]chromone

6-Hydroxy-2-[2-(4'-hydroxy-3'-methoxyphenyl)ethenyl]chromone

2-[2-(4'-Methoxyphenyl)ethyl]chromone

6-Methoxy-2-[2-(4'-methoxyphenyl)ethyl]chromone

7,8-Dimethoxy-2-[2-(3'-acetoxyphenyl)ethyl]chromone

7-Hydroxy-2-(2-phenylethyl)chromone

6-Hydroxy-2-[2-(4-hydroxyphenyl)ethyl]chromone

6,8-Dihydroxy-2-(2-phenylethyl)chromone

6-Hydroxy-7-methoxy-2-(2-phenylethyl)chromone

5-Hydroxy-6-methoxy-2-[2-(3-hydroxy-4-methoxyphenyl)ethyl]chromone

6-Methoxy-2-[2-(3-hydroxy-4-methoxyphenyl)ethyl]chromone

5-Hydroxy-6-methoxy-2-[2-(4-methoxyphenyl)ethyl]chromone

6-Methoxy-2-[2-(4'-hydroxyphenyl)ethyl]chromone

(R)-2-(2-Hydroxy-2-phenylethyl)chromone

(S)-2-(2-Hydroxy-2-phenylethyl)chromone

2-[2-(3-Methoxy-4-hydroxyphenyl)ethyl]chromone (qinanones B)

2-[2-(3-Hydroxy-4-methoxyphenyl)ethyl]chromone (qinanones A)

2-[2-(2-Hydroxy-4-methoxyphenyl)ethyl]chromone (qinanones C)

2 -[2-(4-Hydroxyphenyl)ethyl]chromone (qinanones D)

2-[2-(3-Hydroxyphenyl)ethyl]chromone (qinanones E)

Qinanone $\mathrm{F}$

6-Hydroxy-2-[2-(3-hydroxy-4-methoxyphenyl)ethyl]chromone

5-Hydroxy-6,7-dimethoxy-2-[2-(4'-methoxyphenyl)ethyl]chromone

5-Hydroxyl-7-methoxy-2-[2-(4'-methoxyphenyl)ethyl]chromone

5,8-Dihydroxy-6-methoxy-2-(2-phenylethyl)chromone

6-Methoxy-2-[2-(2', $3^{\prime}, 4^{\prime}$-trihydroxy)phenylethyl]chromone

6-Hydroxy-7-methoxy-2-[2-(4-methoxyphenyl)ethyl]chromone

6-Hydroxy-2-[2-(3,4-dimethoxyphenyl)ethyl]chromone

6,8-Dihydroxy-2-[2-(4-methoxyphenyl)ethyl]chromone

8-Chloro-6-hydroxy-2-[2-(3-methoxy-4-hydroxyphenyl)ethyl]chromone

5-Methoxy-6-hydroxy-2-[2-(3-methoxy-4-hydroxyphenyl)ethyl]chromone

(R)-6,7-Dimethoxy-2-(2-hydroxy-2-phenylethyl)chromone

(S)-6,7-Dimethoxy-2-(2-hydroxy-2-phenylethyl)chromone

7-Methoxy-2-[2-(4'-hydroxy-phenyl)ethyl]chromone

7-Hydroxy-2-[2-(4'-methoxyphenyl)ethyl]chromone

5,6-Dihydroxy-2-[2-(3'-hydroxy-4'-methoxyphenyl)ethyl]chromone

6-Hydroxy-5-methoxy-2-(2-phenyl-ethyl)chromone

\begin{tabular}{|c|c|c|}
\hline $\begin{array}{l}51,65,76,84 \text { and } \\
102\end{array}$ & 33,63 and 99 & - \\
\hline $\begin{array}{l}65,76,79 * \text { and } \\
102\end{array}$ & 33 and 86 & 103 \\
\hline 65 and 102 & 33 and 86 & - \\
\hline 65,76 and $79^{*}$ & - & - \\
\hline 65 and $79^{*}$ & 50 and 86 & - \\
\hline $\begin{array}{l}51,65,79^{*} \text { and } \\
102\end{array}$ & 33 and 86 & - \\
\hline $\begin{array}{l}66 \text { and } 84 \\
66\end{array}$ & 91 & - \\
\hline $48^{*}, 51$ and 66 & 33 and 91 & - \\
\hline 67 and $79^{*}$ & 80 and $99^{*}$ & - \\
\hline 67 & - & - \\
\hline 68 and $80^{*}$ & - & - \\
\hline 76 and 104 & - & - \\
\hline 104 & - & - \\
\hline 73,75 and $80^{*}$ & - & - \\
\hline 73 and $80^{*}$ & - & - \\
\hline 73 & - & - \\
\hline 73 & - & - \\
\hline 73 and $80^{*}$ & - & - \\
\hline 73 & - & - \\
\hline 73 and 75 & - & - \\
\hline 73 and 76 & - & - \\
\hline 76,84 and 105 & 50 and 90 & - \\
\hline $79^{*}$ & 50 and 90 & - \\
\hline- & 98 & - \\
\hline- & 99 & - \\
\hline- & 99 & - \\
\hline $79 *$ & 99 & - \\
\hline 51 & 99 & - \\
\hline $79 *$ & - & - \\
\hline $79^{*}$ and $80^{*}$ & - & - \\
\hline $79^{*}$ & - & - \\
\hline $79 *$ & - & - \\
\hline- & - & 103 \\
\hline- & - & 103 \\
\hline 76 & - & 103 \\
\hline 76 & - & - \\
\hline 76 & - & 103 \\
\hline 76 & - & - \\
\hline 76 & - & - \\
\hline 76 & - & - \\
\hline 76 and $80^{*}$ & - & - \\
\hline 84 & - & - \\
\hline $83^{*}$ & - & - \\
\hline $83^{*}$ & - & - \\
\hline $83 *$ & - & - \\
\hline $80^{*}$ & - & - \\
\hline $80^{*}$ & - & - \\
\hline $80^{*}$ & - & - \\
\hline $80^{*}$ & - & - \\
\hline $80^{*}$ & - & - \\
\hline $80^{*}$ & - & - \\
\hline $80^{*}$ & - & - \\
\hline 51 & - & - \\
\hline 51 & - & - \\
\hline 51 & - & - \\
\hline 51 & - & - \\
\hline
\end{tabular}




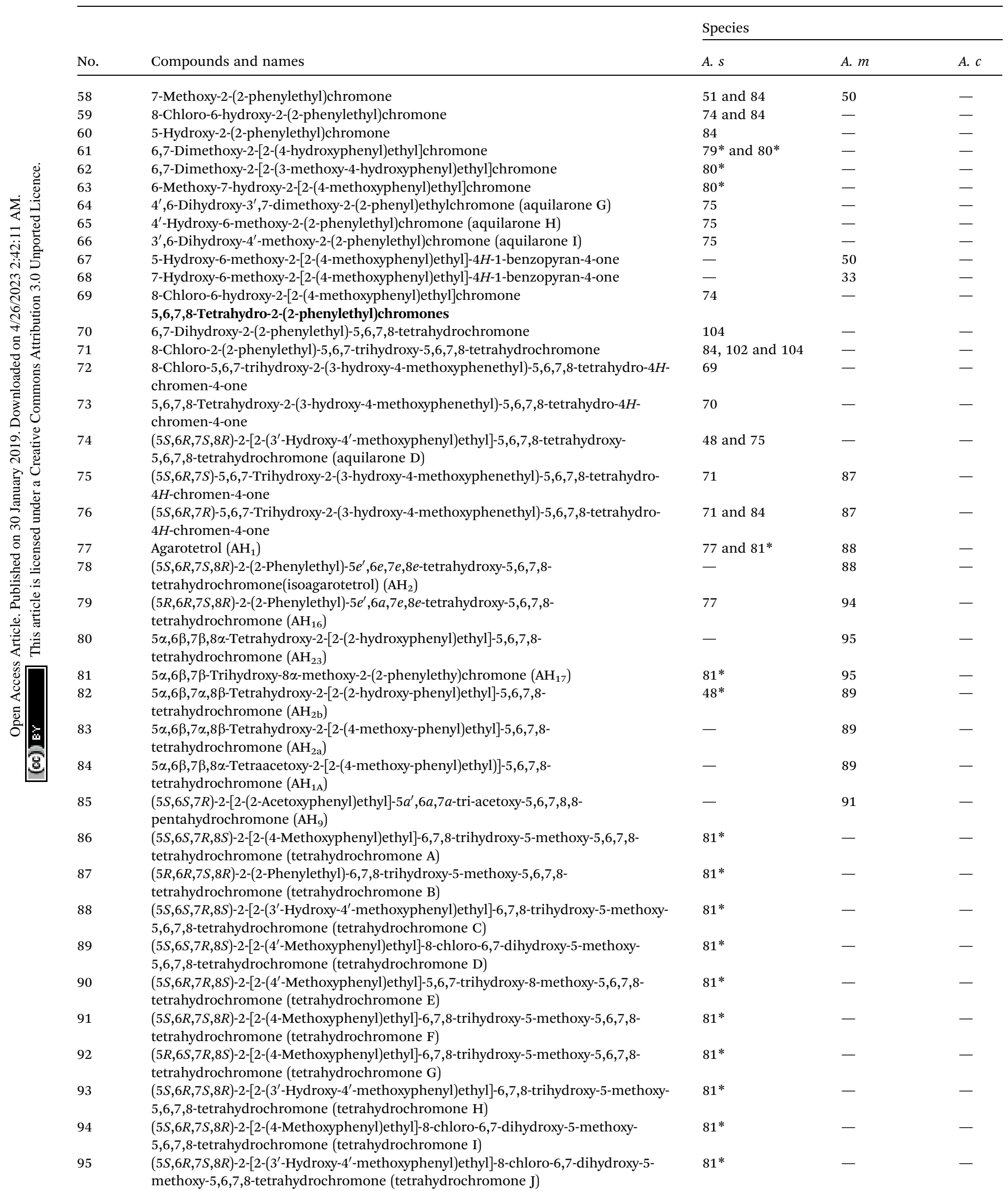




\begin{tabular}{|c|c|c|c|c|}
\hline \multirow[b]{2}{*}{ No. } & \multirow[b]{2}{*}{ Compounds and names } & \multicolumn{3}{|l|}{ Species } \\
\hline & & A. $s$ & A. $m$ & A. $c$ \\
\hline 96 & $\begin{array}{l}(5 S, 6 R, 7 R, 8 S)-2-\left[2-\left(4^{\prime}-\text { Methoxyphenylethyl }\right)\right]-5,6,7,8 \text {-tetrahydroxy-5,6,7,8- } \\
\text { tetrahydrochromone }\end{array}$ & $81^{*}$ & - & - \\
\hline 97 & $\begin{array}{l}\text { rel-(5R,6S,7S,8R)-8-Chloro-5,6,7,8-tetrahydro-5,6,7-trihydroxy-2-[2-(4- } \\
\text { methoxyphenyl)ethyl]-4H-1-benzopyran-4-one }\end{array}$ & $81^{*}$ & 33 & - \\
\hline 98 & $\begin{array}{l}(5 S, 6 S, 7 S, 8 R)-2-\left[2-\left(3^{\prime}-\text { Hydroxy-4'-methoxyphenyl)ethyl]-5,6,7,8-tetrahydroxy-5,6,7,8- }\right.\right. \\
\text { tetrahydrochromone (aquilarone A) }\end{array}$ & 75 and $81^{*}$ & - & - \\
\hline 99 & $\begin{array}{l}\text { (5S,6S,7S,8R)-2-(2-Phenylethyl)-5,6,7,8-tetrahydroxy-5,6,7,8-tetrahydrochromone } \\
\text { (aquilarone B) }\end{array}$ & $75,81^{*}$ and 84 & - & - \\
\hline 100 & $\begin{array}{l}(5 S, 6 S, 7 S, 8 R)-2-\left[2-\left(4^{\prime} \text {-Methoxyphenyl)ethyl]-5,6,7,8-tetrahydroxy-5,6,7,8- }\right.\right. \\
\text { tetrahydrochromone (aquilarone C) }\end{array}$ & $75,81^{*}$ and 84 & - & - \\
\hline 101 & $\begin{array}{l}(5 S, 6 R, 7 R, 8 S)-2-\left[2-\left(3^{\prime} \text {-Hydroxy-4'-methoxyphenyl)ethyl]-5,6,7,8-tetrahydroxy- }\right.\right. \\
5,6,7,8 \text {-tetrahydrochromone (aquilarone E) }\end{array}$ & 75 and $81^{*}$ & - & - \\
\hline 102 & $\begin{array}{l}\text { (5R,6R,7R,8S)-8-Chloro-5,6,7-trihydroxy-2-(4-methoxyphenethyl)-5,6,7,8- } \\
\text { tetrahydrochromone }\end{array}$ & 84 & - & - \\
\hline 103 & $\begin{array}{l}(5 S, 6 S, 7 S, 8 S) \text {-8-Chloro-5,6,7-trihydroxy-2-(2-phenylethyl)-5,6,7,8- } \\
\text { tetrahydrochromone }\end{array}$ & 84 & - & - \\
\hline 104 & $\begin{array}{l}\text { (5R,6R,7R,8R)-8-Chloro-5,6,7-trihydroxy-2-(4-methoxyphenethyl)-5,6,7,8- } \\
\text { tetrahydrochromone }\end{array}$ & 84 & - & - \\
\hline 105 & $\begin{array}{l}(5 R, 6 S, 7 S)-5,6,7-T r i h y d r o x y-2-(4 \text {-hydroxy-3-methoxyphenethyl)-5,6,7,8- } \\
\text { tetrahydrochromone }\end{array}$ & 84 & - & - \\
\hline 106 & $\begin{array}{l}(5 S, 6 R, 7 R, 8 S)-2-\left[2-\left(4^{\prime}-\text { Hydroxyphenyl)ethyl]-5,6,7,8-tetrahydroxy-5,6,7,8- }\right.\right. \\
\text { tetrahydrochromone (aquilarone F) }\end{array}$ & 75 & - & - \\
\hline 107 & $\begin{array}{l}\text { rel-(5R,6S,7S,8R)-8-Chloro-5,6,7,8-tetrahydro-5,6,7-trihydroxy-2-[2-(3-hydroxy-4- } \\
\text { methoxyphenyl)ethyl]-4H-1-benzopyran-4-one }\end{array}$ & - & 33 & - \\
\hline 108 & $\begin{array}{l}\text { rel-(5R,6S,7R)-5,6,7,8-Tetrahydro-5,6,7-trihydroxy-2-(2-phenylethyl)-4H-1- } \\
\text { benzopyran-4-one }\end{array}$ & - & 33 & - \\
\hline 109 & $\begin{array}{l}\text { rel-(5R,6S,7R)-5,6,7,8-Tetrahydro-5,6,7-trihydroxy-2-[2-(4-methoxyphenyl)ethyl]-4H- } \\
\text { 1-benzopyran-4-one } \\
\text { Diepoxy-tetrahydro-2-(2-phenylethyl)chromones }\end{array}$ & - & 33 & - \\
\hline 110 & $\begin{array}{l}\text { 5,6:7,8-Diepoxy-2-(2-phenylethyl)-5,6,7,8-tetrahydrochromone } \\
\text { (oxidoagarochromone A) }\end{array}$ & $72^{*}, 79^{*}$ and $81^{*}$ & 33 & $72 *$ \\
\hline 111 & $\begin{array}{l}\text { 5,6:7,8-Diepoxy-2-[2-(4-methoxyphenyl)ethyl]-5,6,7,8-tetrahydrochromone } \\
\text { (oxidoagarochromone B) }\end{array}$ & $72 *$ and $79 *$ & 33 & $72^{*}$ \\
\hline 112 & $\begin{array}{l}\text { 5,6:7,8-Diepoxy-2-[2-(3-hydroxy-4-methoxyphenyl)ethyl]-5,6,7,8- } \\
\text { tetrahydrochromone (oxidoagarochromone C) }\end{array}$ & $72^{*}$ & 33 & $72^{*}$ \\
\hline 113 & 5,6-Epoxy-7 $\beta$-hydroxy-8 $\beta$-methoxy-2-(2-phenylethyl)chromone & $79^{*}$ & - & - \\
\hline 114 & $\begin{array}{l}\text { (5S,6R,7R,8R)-2-(2-Phenylethyl)-7,8-epoxy-5,6-dihydroxy-5,6,7,8-tetrahydrochrome } \\
\text { (tetrahydrochromone K) }\end{array}$ & $81^{*}$ & - & - \\
\hline 115 & $\begin{array}{l}(5 R, 6 S, 7 S, 8 S)-2-\left[2-\left(4^{\prime}-\text { Methoxyphenyl)ethyl]-7,8-epoxy-5,6-dihydroxy-5,6,7,8- }\right.\right. \\
\text { tetrahydrochrome (tetrahydrochromone L) }\end{array}$ & $81^{*}$ & - & - \\
\hline 116 & $\begin{array}{l}(5 R, 6 S, 7 S, 8 S)-2-\left[2-\left(3^{\prime} \text {-Hydroxy-4'-methoxyphenyl)ethyl]-7,8-epoxy-5,6-dihydroxy- }\right.\right. \\
5,6,7,8 \text {-tetrahydrochrome (tetrahydrochromone } \mathrm{M})\end{array}$ & $81^{*}$ & - & - \\
\hline 117 & $5 \alpha, 6 \alpha$-Epoxy-7 $\beta, 8 \alpha, 3^{\prime}$-trihydroxy-4'-methoxy-2-(2-phenylethyl)chromone & $83^{*}$ & - & - \\
\hline 118 & $\begin{array}{l}\text { rel-(1aR,2R,3R,7bS)-1a,2,3,7b-Tetrahydro-2,3-dihydroxy-5-[2-(4-methoxyphenyl) } \\
\text { ethyl]-7H-oxireno[f][1] benzopyran-7-one }\end{array}$ & 84 & 33 & - \\
\hline 119 & $\begin{array}{l}\text { rel-(1aR,2R,3R,7bS)-1a,2,3,7b-Tetrahydro-2,3-dihydroxy-5-(2-phenylethyl)-7H- } \\
\text { oxireno[f][1]benzopyran-7-one }\end{array}$ & $79^{*}$ & 33 & - \\
\hline 120 & Qinanmer & 77 & - & - \\
\hline 121 & 2-[2-(4-Glucosyloxy-3-methoxyphenyl)ethyl]chromone & 78 & - & - \\
\hline 122 & $\begin{array}{l}(5 S, 6 S, 7 R, 8 S)-2-(2-P h e n y l e t h y l)-6,7,8 \text {-trihydroxy-5,6,7,8-tetrahydro-5-[2-(2- } \\
\text { phenylethyl)chromonyl-6-oxy]chromone }\left(\mathrm{AH}_{10}\right)\end{array}$ & - & 92 & - \\
\hline 123 & $\begin{array}{l}\text { (5S,6S,7R,8S)-2-(2-Phenylethyl)-6,7,8-trihydroxy-5,6,7,8-tetrahydro-5-[2-(2- } \\
\text { phenylethyl)-7-hydroxy-chromonyl-6-oxy]chromone }\left(\mathrm{AH}_{15}\right)\end{array}$ & - & 92 & - \\
\hline 124 & 2,2'-Di-(2-phenylethyl)-8, $6^{\prime}$-dihydroxy-5,5'-bichromone $\left(\mathrm{AH}_{11}\right)$ & - & 92 & - \\
\hline 125 & $\begin{array}{l}(5 S, 6 R, 7 R, 8 S)-2-(2-\text {-Phenylethyl)-5,6,7-trihydroxy-5,6,7,8-tetrahydro-8-[2-(2- } \\
\text { phenylethyl)-7-methoxychromonyl-6-oxy]chromone }\left(\mathrm{AH}_{12}\right)\end{array}$ & - & 92 & - \\
\hline 126 & $\begin{array}{l}(5 S, 6 R, 7 R, 8 S)-2-(2-\text { Phenylethyl)-5,6,7-trihydroxy-5,6,7,8-tetrahydro-8-[2-(2- } \\
\text { phenylethyl)chromonyl-6-oxy]chromone }\left(\mathrm{AH}_{13}\right)\end{array}$ & - & 92 & - \\
\hline 127 & $\begin{array}{l}(5 S, 6 S, 7 S, 8 R)-2-(2-P h e n y l e t h y l)-6,7,8 \text {-trihydroxy-5,6,7,8-tetrahydro-5-[2-(2- } \\
\text { phenylethyl)-chromonyl-6-oxy]chromone }\left(\mathrm{AH}_{14}\right)\end{array}$ & - & 92 & - \\
\hline 128 & Dioxin-linked bi-2-(2-phenylethyl)chromone $\left(\mathrm{AH}_{21}\right)$ & - & 97 & - \\
\hline
\end{tabular}




\begin{tabular}{|c|c|c|c|c|}
\hline \multirow[b]{2}{*}{ No. } & \multirow[b]{2}{*}{ Compounds and names } & \multicolumn{3}{|c|}{ Species } \\
\hline & & A. $s$ & A. $m$ & A. $c$ \\
\hline 129 & $\begin{array}{l}\text { Bi- }(5 S, 6 S, 7 R, 8 S) \text {-2-(2-phenylethyl)-6,7,8-trihydroxy-5,6,7,8-tetrahydro-5-[2-(2- } \\
\text { phenylethyl)chromonyl-6,7-dioxy]chromone }\left(\mathrm{AH}_{18}\right)\end{array}$ & - & 93 & - \\
\hline 130 & AH19a & - & 96 & - \\
\hline 131 & AH19b & - & 96 & - \\
\hline 132 & $\mathrm{AH} 20$ & - & 95 & - \\
\hline 133 & $\begin{array}{l}(5 S, 6 R, 7 S, 8 R)-2-[2-(4-M e t h o x y p h e n y l) e t h y l]-5,6,7-t r i h y d r o x y-5,6,7,8 \text {-tetrahydro-8-\{6- } \\
\text { methoxy-2-[2-(3'"'-methoxy-4"'-hydroxyphenyl)ethyl]chromonyl-7-oxy\}chromone }\end{array}$ & $82^{*}$ & - & - \\
\hline 134 & $\begin{array}{l}(5 S, 6 R, 7 S, 8 R)-2-[2-(4-M e t h o x y p h e n y l) e t h y l]-5,6,7 \text {-trihydroxy-5,6,7,8-tetrahydro-8- }\{2- \\
{\left[2-\left(4^{\prime \prime \prime} \text {-methoxyphenyl)ethyl]chromonyl-6-oxy }\right\} \text { chromone }\right.}\end{array}$ & $82 *$ & - & - \\
\hline 135 & $\begin{array}{l}(5 S, 6 R, 7 S, 8 R)-2 \text {-(2-Phenylethyl)-5,6,7-trihydroxy-5,6,7,8-tetrahydro-8-[2-(2- } \\
\text { phenylethyl)chromonyl-6-oxy]chromone }\end{array}$ & $82 *$ & - & - \\
\hline 136 & $\begin{array}{l}(5 R, 6 R, 7 R, 8 S)-2 \text {-(2-Phenylethyl)-5,6,7-trihydroxy-5,6,7,8-tetrahydro-8-[2-(2- } \\
\text { phenylethyl)chromonyl-6-oxy]chromone }\end{array}$ & $82 *$ & - & - \\
\hline 137 & Crassin A & - & - & 100 \\
\hline 138 & $(5 R, 6 S, 7 R, 8 S)$-Configuration (crassin $\mathrm{B})$ & - & - & 100 \\
\hline 139 & $(5 S, 6 R, 7 S, 8 R)$-Configuration (crassin $\mathrm{C})$ & - & - & 100 \\
\hline 140 & Crassin D & - & - & 100 \\
\hline 141 & Aquilacrassnin A & - & - & 101 \\
\hline 142 & Aquilacrassnin B & - & - & 101 \\
\hline 143 & Aquilacrassnin $\mathrm{C}$ & - & - & 101 \\
\hline 144 & Aquilacrassnin D & - & - & 101 \\
\hline 145 & Aquilacrassnin E & - & - & 101 \\
\hline 146 & Aquilacrassnin $\mathrm{F}$ & - & - & 101 \\
\hline 147 & $(5 S, 6 R, 7 S, 8 R)-(+)$-Aquisinenone A & 85 & - & - \\
\hline 148 & $(5 R, 6 S, 7 R, 8 S)-(-)$-Aquisinenone A & 85 & - & - \\
\hline 149 & $(5 R, 6 S, 7 R, 8 S)-(-)-4^{\prime}-$ Methoxyaquisinenone A & 85 & - & - \\
\hline 150 & $(5 R, 6 S, 7 R, 8 S)-(+)$-Aquisinenones $\mathrm{B}$ & 85 & - & - \\
\hline 151 & $(5 S, 6 R, 7 S, 8 R)-(-)$-Aquisinenones B & 85 & - & - \\
\hline 152 & $(5 S, 6 R, 7 S, 8 R)-(-)-6^{\prime \prime}$-Hydroxyaquisinenone B & 85 & - & - \\
\hline 153 & $(5 R, 6 S, 7 R, 8 S)-(+)-6^{\prime \prime}-$ Hydroxy- $4^{\prime}, 4^{\prime \prime \prime}$-dimethoxyaquisinenone B & 85 & - & - \\
\hline 154 & $(5 R, 6 S, 7 R, 8 S)-(+)$-Aquisinenones $\mathrm{C}$ & 85 & - & - \\
\hline 155 & $(5 S, 6 R, 7 S, 8 R)-(-)$-Aquisinenones $\mathrm{C}$ & 85 & - & - \\
\hline 156 & $(5 S, 6 R, 7 S, 8 R)-(-)$-Aquisinenone $\mathrm{D}$ & 85 & - & - \\
\hline 157 & $(5 R, 6 S, 7 R, 8 S)-4^{\prime}$-Demethoxyaquisinenone D & 85 & - & - \\
\hline 158 & $(5 S, 6 R, 7 S, 8 R)-4^{\prime}$-Demethoxyaquisinenone D & 85 & - & - \\
\hline 159 & $(5 S, 6 R, 7 S, 8 R)-(+)$-Aquisinenone $\mathrm{E}$ & 85 & - & - \\
\hline 160 & $(5 S, 6 R, 7 S, 8 R)-(-)$-Aquisinenone $\mathrm{F}$ & 85 & - & - \\
\hline 161 & $(5 S, 6 R, 7 S, 8 R)-(-)$-Aquisinenone $\mathrm{G}$ & 85 & - & - \\
\hline 162 & (+)-4'-Methoxyaquisinenone G & 85 & - & - \\
\hline
\end{tabular}

${ }^{a}$ A. s, A. $m$, and A. $c$ indicate A. sinensis, A. malaccensis, and A. crassna, respectively. ${ }^{b}$ The reference was not found. ${ }^{c}$ "*” indicates that the agarwood in this article was artificial agarwood.

(Gramineae), and agarwoods originating from Aquilaria spp. (Thymelaeaceae). 2-(2-Phenylethyl)chromone derivatives are the characteristic components of agarwoods, and more than 40 such derivatives have been found in agarwoods belonging to different species. Depending on the molecular skeleton, chromones can be divided into 2-(2-phenylethyl)chromones, 5,6,7,8tetrahydro-2-(2-phenylethyl)chromones, diepoxy-tetrahydro-2(2-phenylethyl)chromones, and associated chromones (shown in Scheme 2). As shown in Table 3, the variation of chromones in different species is striking.

Regarding the study of chromones, most researchers use agarwood extracts, usually ethanol (EtOH) extracts, to extract and separate the monomers. The structures of the compounds are determined by a series of assays, including LC/MS, and nuclear magnetic resonance.

\subsection{Chromones in A. sinensis}

Approximately 130 chromone derivatives have been obtained from $A$. sinensis, comprising 22 forms of 2-(2-phenylethyl)chromones, six 5,6,7,8-tetrahydro-2-(2-phenylethyl)chromones, and three diepoxy-tetrahydro-2-(2-phenylethyl)chromones.

Yang et al. obtained 1-6 from an EtOH extract of $A$. sinensis, which belong to the group of 2-(2-phenylethyl)chromones; ${ }^{65}$ they later extracted 7-9 from an EtOAc-EtOH extract. ${ }^{66}$ In addition, 10-12 were isolated by Liu et al. ${ }^{67,68}$ Dai et al. extracted 165-168 from the same species. ${ }^{69-71}$ Yagura and coworkers obtained four chromones, 13, 14, 70 and 71, in 2003 and later extracted 110-112;72 these are all diepoxy tetrahydrochromones. In 2012, Yang and coworkers isolated eight new chromone derivatives, $15-22 .^{73}$ Gao et al. ${ }^{74}$ and Chen et al. ${ }^{75}$ isolated 59, 69, and aquilarones A-I (64-66, 74, 98-101, 106), 
with two known chromones, 15 and 21, from an EtOH extract of resinous wood of $A$. sinensis. Yang ${ }^{76}$ obtained 2-(2-phenylethyl) chromone derivatives 1, 2, 4, 13, 23, 22, and 36-42 from a $\mathrm{Et}_{2} \mathrm{O}$ extract of "Qi-Nan". Later, this research team ${ }^{77,78}$ found a new compound, 120, comprising 2-(2-phenylethyl)chromone and sesquiterpene moieties, named “Qinanmer”; a 2-(2-phenylethyl) chromone glycoside, 121, together with two 2-(2-phenylethyl) chromone derivatives, 77 and 79, were obtained from a EtOH extract of "Qi-Nan".

Since 2014, researchers have been engaged in the study of artificial agarwood induced by the holing method. Li et al. ${ }^{79}$ isolated three previously undescribed 2-(2-phenylethyl)chromone derivatives, 30,31 , and 113 , and thirteen 5,6,7,8tetrahydro-2-(2-phenylethyl)chromones, named tetrahydrochromones A-M (86-95, 114-116), together with thirteen known ones $(2,4-6,10,24,28,32,33,110,111,118$, and 119) from an EtOAC extract. Liao et al. ${ }^{\mathbf{8 0 , 8 1}}$ used the same method and found 2-(2-phenylethyl)chromone derivatives 12, 15, 16, 19, 42, 47-53, 61-63, 77, 81, 96-101, and 110. The EtOAc fraction also contained four new bi-phenylethylchromones, 133-136. ${ }^{\mathbf{8 2}}$ Kuang et $a .^{48}$ were also interested in agarwood induced by artificial holing; they researched the chemical constituents of the $n$-butanol fraction of an EtOH extract and obtained 9, 74, and 82 .

Liu et $a l .{ }^{83}$ separated and identified 44-46 and 117 from an EtOH extract of agarwood produced via the whole-tree agarwood-inducing technique.

Huo and coworkers ${ }^{84}$ isolated 2-(2-phenylethyl)chromone derivatives $1,7,23,43,58-60,71,76,99,100,102-105$, and 118 from a 95\% EtOH-EtOAc extract of resinous wood of A. sinensis. Subsequently, through LC-MS-guided separation and purification, they obtained sixteen new 2-(2-phenylethyl)chromone dimers, including four pairs of enantiomers, along with eight optically pure analogues (151-162). ${ }^{85}$ Wang et al. ${ }^{51}$ isolated compounds 54-57, which belong to the group of 2-(2-phenylethyl)chromone derivatives, from resinous wood, together with five known compounds, 1, 6, 9, 29, and 58, from a $\mathrm{MeOH}$ extract.

\subsection{Chromones in A. malaccensis (or A. agallocha)}

More than 30 chromones have been reported from A. agallocha, of which nine are the same as in $A$. sinensis, namely $1,{ }^{63} 2,3{ }^{86} 5$, $6,{ }^{86} 9,^{87}$ and 71 and $72 .{ }^{87}$ Since 1982 , Shimada and coworkers have been engaged in the isolation of chromones 2-3, 5-6, 77$78,{ }^{88} 82,{ }^{89}$ and $83 .{ }^{89}$ In 1986 , Nakanishi isolated a known chromone, 23, and a new chromone, $24 .{ }^{90}$ Then, $7,9,85,{ }^{91} 122-127,{ }^{92}$ and 129 (ref. 93) were isolated and identified. Konishi devoted himself to this work, also aiding other researchers in the field; from 1989 to 1992 , he found $79,{ }^{94} 80,81,132,{ }^{95} 130-131,{ }^{96} 128,{ }^{97}$ and $71-72 .{ }^{87}$ Iwagoe obtained 123 and $129,,^{93}$ and in 2005, Alkhathlan isolated 3, 6, and 25 from A. agallocha. ${ }^{98}$

The chromones isolated from A. malaccensis were mainly reported by $\mathrm{T}$. Konishi in 2002, namely $1,26-29$, and $10 .{ }^{99} \mathrm{Wu}$ et $a .^{33,50}$ reported the 2-(2-phenylethyl)-4H-chromone derivatives $1-3,5,6,9,14,23,24,58,67,68,97,107-112,118$, and 119 from a $70 \% \mathrm{MeOH}$ extract of $A$. malaccensis agarwood.

\subsection{Chromones in A. crassna}

There are few reports on chromones in A. crassna. Diepoxytetrahydro-2-(2-phenylethyl) chromones 110-112 were obtained from $A$. crassna. ${ }^{72}$ Yang et al. ${ }^{\mathbf{1 0 0 , 1 0 1}}$ obtained four new bi-2(2-phenylethyl)chromone derivatives, crassins A-D (137-140), and six previously undescribed uncommon ester-bonded dimeric compound aquilacrassnins A-F (141-146) from the EtOAc extract of agarwood originating from A. crassna.

\section{Discussion}

Among the 367 new main chemical constituents from agarwoods that were statistically assessed in this paper, chromone derivatives and sesquiterpenes accounted for $44.14 \%$ and $55.86 \%$, respectively, of the total constituents. It can be seen in Fig. 1(a) that the largest numbers of sesquiterpenes in agarwood are eudesmanes, guaianes and eremophilanes. Fig. 1(b) reflects the number of different chromones in agarwood, where 2-(2-

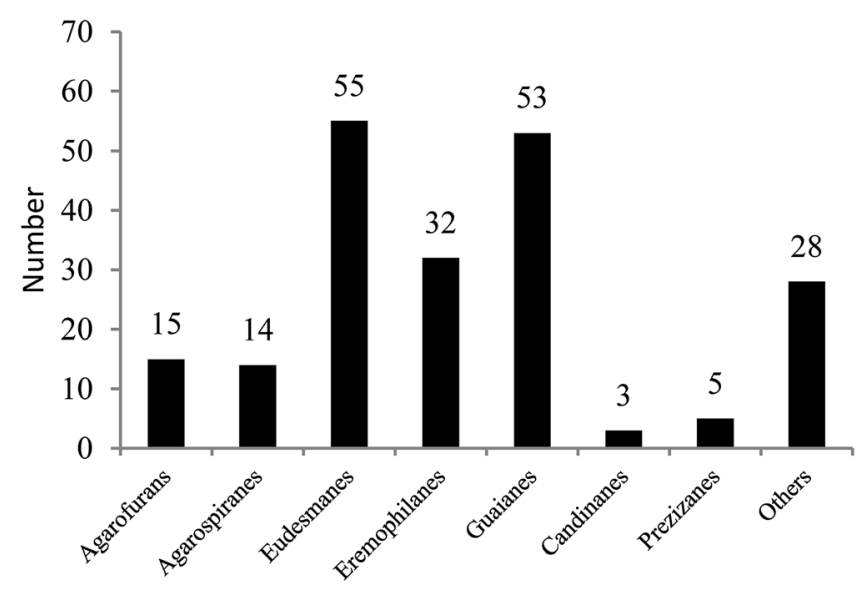

(a)

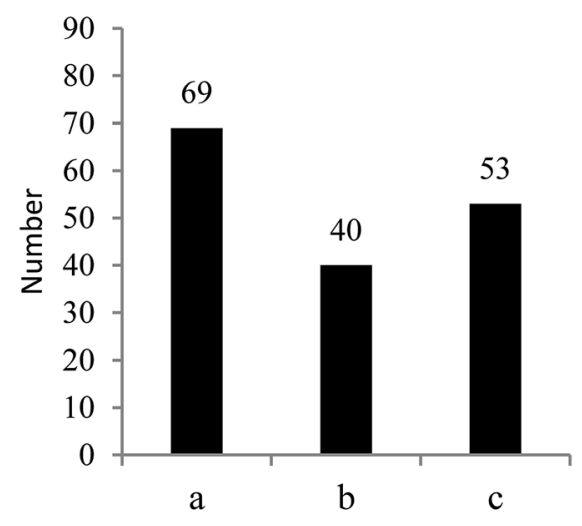

(b)

Fig. 1 (a) The number of different types of sesquiterpenes in agarwood; (b) the number of different types of chromone derivatives in agarwood ((a) 2-(2-phenylethyl)chromones; (b) 5,6,7,8-tetrahydro-2-(2-phenylethyl)chromones; (c) others). 


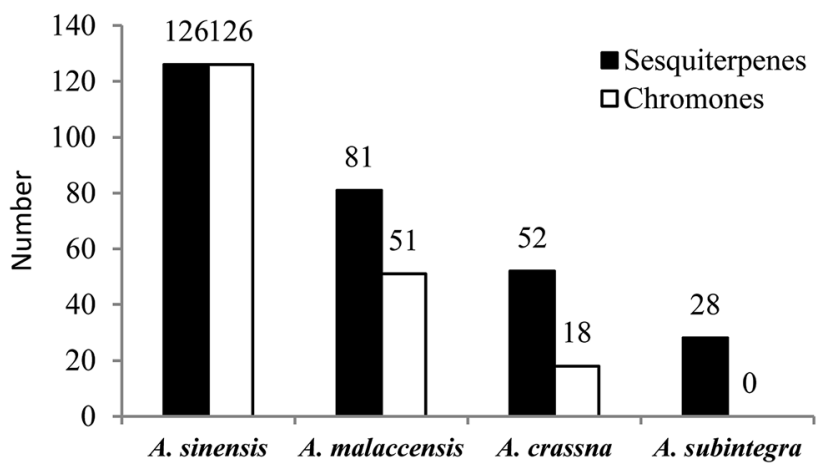

Fig. 2 The numbers of sesquiterpenes and chromone derivatives in different species of agarwood.

phenylethyl)chromones are currently the most commonly isolated types.

From the statistical results (shown in Fig. 2), researchers are currently mainly engaged in the study of the chemical constituents of agarwood originating from A. sinensis, A. malaccensis, and A. crassna, respectively, of which most of the new compounds were isolated from $A$. sinensis. It can be seen that resources are important prerequisites for the study of agarwood; thus, there are many studies on species with relatively abundant resources, such as A. sinensis, A. malaccensis, and A. crassna. Of course, this is also closely related to geographical distribution. Agarwoods originating from different Aquilaria plants contain some common compounds as well as some different compounds. Among different species of agarwood, the chemical compositions are quite different. Therefore, it is necessary to indicate the species from which the used agarwood is derived. However, during the writing process, we found that many articles on the separation of compounds from agarwood did not indicate which species of the genus Aquilaria the agarwood was derived from. Therefore, we encourage researchers studying agarwood to indicate more information about the origin and tree species to clarify the source of the material.

According to the data, the number of sesquiterpenes isolated from agarwood has thus far been higher than the number of chromones, and the proportion of articles is also the same. In the past 10 years, the number of articles on chromones has increased rapidly. The study of the chemical constituents of agarwood from sesquiterpenes to chromone derivatives shows that increasing numbers of researchers are beginning to focus on revealing the main components of agaric pharmacologically active substances rather than only fragrance components. Therefore, we can see that research on the separation and activity of chromone derivatives still has broad research prospects.

By summarizing and comparing the chemical compositions of different tree species, we can provide more research ideas. The same components can be used as standards for quality assessment, with reliable and stable characteristics, and different components can guide the selection of high quality agarwood species. By reviewing the chemical compositions of agarwoods from the four species, we believe that the following characteristics should be considered when selecting quality control standards. Due to the complex composition of agarwood, sesquiterpenes and chromone derivatives should be considered first, especially chromones, mainly because they are characteristic components of agarwood, and chromone derivatives are easier to separate and preserve. Due to the extremely complex sources and types of agarwood, researchers in different countries should fully consider the common chemical composition when selecting control indicators and formulating quality testing methods to improve the scope and scientificity of the testing methods, such as F2 and F3. Of course, even with the limited amounts of research on individual species, it is possible to flexibly select components, such as chromone 1 .

\section{Conclusion}

Agarwood, which is expensive and widely used, is derived from the resin-containing wood of Aquilaria species trees. The chemical components of agarwood are diverse and complex; 367 new chemical constituents from agarwood were statistically assessed in this paper. This review summarizes the main molecular skeletons of agarwood compounds, revealing the differences in the chemical compositions of agarwood originating from different Aquilaria species. This will help researchers to better understand research on agarwood and select more suitable detection indicators.

With the continuous exploration and efforts made by scientists in recent years, the understanding of the chemical compositions of agarwood from different sources is continuously improving, and some specific chemical compositions may become identification indices and judgement standards of agarwood samples from different sources. In the future, we expect to see more research on the chemical components of agarwood from different species in order to help identify characteristic compounds of agarwood, establish a stable, effective, comprehensive, and reliable quality evaluation system, and consequently elucidate which species best produce agarwood.

\section{Conflicts of interest}

The authors declare no conflict of interest.

\section{Acknowledgements}

This work was supported by the National Natural Science Foundation of China (81773844, 31000136), Beijing Municipal Natural Science Foundation (6102024).

\section{References}

1 J. S. Yang, Nat. Prod. Res. Dev., 1998, 10, 99-103.

2 H. Q. Chen, Y. Yang, J. Xue, J. H. Wei, Z. Zhang and H. J. Chen, Molecules, 2011, 16, 4884-4896.

3 M. C. M. Eurlings and B. Gravendeel, Plant Syst. Evol., 2005, 254, 1-12.

4 R. Naef, Flavour Fragrance J., 2011, 26, 73-87. 
5 The Plant List, http:/www.theplantlist.org/tpl1.1/search? q=Aquilaria, accessed December 6, 2018.

6 Checklist of CITES species, http://checklist.cites.org/\#/en, accessed December 6, 2018.

7 CITES Secretariat and Missouri Botanical Garden, http:// www.tropicos.org/, accessed December 6, 2018.

8 Y. Z. H. Y. Hashim, P. G. Kerr, P. Abbas and H. M. Salleh, J. Ethnopharmacol., 2016, 189, 331-360.

9 K. Jayachandran, I. Sekar, K. T. Parthiban, D. Amirtham and K. K. Suresh, Indian J. Nat. Prod. Resour., 2014, 1, 44-47.

10 M. Saikia, K. Shrivastava and S. S. Singh, Int. J. Plant Sci., 2012, 2, 188-194.

11 Y. B. Li, Doctor thesis, Guangzhou University of Chinese Medicine, 2017.

12 J. Yang, Y. L. Wang, Y. L. Su, C. H. He, Q. T. Zheng and J. Yang, Acta Pharmacol. Sin., 1989, 264-268.

13 W. L. Mei, Y. B. Zeng, J. Wu, H. B. Cui and H. F. Dai, J. Chin. Pharm. Sci., 2008, 17, 225-229.

14 T. Nakanishi, E. Yamagata, K. Yoneda, T. Nagashima, I. Kawasaki, T. Yoshida, H. Mori and I. Miura, Phytochemistry, 1984, 23, 2066-2067.

15 M. L. Maheshwari, T. C. Jain, R. B. Bates and S. C. Bhattacharyya, Tetrahedron, 1963, 19, 1079-1090.

16 P. Pripdeevech, W. Khummueng and S. K. Park, J. Essent. Oil Res., 2011, 23, 46-53.

17 J. F. Xu, L. F. Zhu, B. Y. Lu and C. T. Liu, Acta Bot. Sin., 1988, 6, 638.

18 T. Nagashima, I. Kawasaki, T. Yoshida, T. Nakanishi and K. Yoneda, New sesquiterpenoids from agarwood, Singapore, 1983, vol. 3, pp. 12-16.

19 R. Näf, A. Velluz, W. Thommen, R. Brauchli, C. Sigwart and J. M. Gaudin, Flavour Fragrance J., 1993, 8, 307-313.

20 M. L. Maheshwari, K. R. Varma and S. C. Bhattacharyya, Tetrahedron, 1963, 19, 1519-1525.

21 J. S. Yang and Y. W. Chen, Acta Pharmacol. Sin., 1986, 516520.

22 J. S. Yang, Y. L. Wang and Y. L. Su, Chin. Chem. Lett., 1992, 983-984.

23 H. X. Huo, Z. X. Zhu, D. R. Pang, Y. T. Li, Z. Huang, S. P. Shi, J. Zheng, Q. Zhang, Y. F. Zhao and P. F. Tu, Fitoterapia, 2015, 106, 115-121.

24 R. Näf, A. Velluz, N. Busset and J. M. Gaudin, Flavour Fragrance J., 1992, 7, 295-298.

25 R. Näf, A. Velluz, R. Brauchli and W. Thommen, Flavour Fragrance J., 1995, 10, 147-152.

26 D. L. Yang, H. Wang, Z. K. Guo, W. Li, W. L. Mei and H. F. Dai, Phytochem. Lett., 2014, 8, 121-125.

27 J. S. Yang and Y. W. Chen, Acta Pharmacol. Sin., 1983, 18, 191-198.

28 F. Lin, H. F. Dai, H. Wang and W. L. Mei, Lishizhen Med. Mater. Med. Res., 2010, 1901-1902.

29 M. L. Maheshwari, S. C. Bhattacharyya and K. R. Varma, Tetrahedron, 1965, 21, 115-138.

30 T. Nakanishi, E. Yamagata, K. Yoneda, I. Miura and H. Mori, J. Chem. Soc., Perkin Trans. 1, 1983, 14, 601-604.

31 T. Nakanishi, E. Yamagata, K. Yoneda and I. Miura, Phytochemistry, 1981, 20, 1597-1599.
32 H. Zhao, Q. Peng, Z. Han, L. Yang and Z. Wang, Molecules, 2016, 21, 281.

33 B. Wu, S. W. Kwon, G. S. Hwang and J. H. Park, Helv. Chim. Acta, 2012, 95, 1657-1665.

34 M. Ishihara, T. Tsuneya, M. Shiga and K. Uneyama, Phytochemistry, 1991, 30, 563-566.

35 J. ya Ueda, L. Imamura, Y. Tezuka, Q. L. Tran, M. Tsuda and S. Kadota, Bioorg. Med. Chem., 2006, 14, 3571-3574.

36 J. J. Tian, X. L. Guo, L. C. Lai, H. B. Zhou, W. M. Zhang and X. X. Gao, Lishizhen Med. Mater. Med. Res, , 2009, 20, 25052507.

37 D. L. Yang, J. Wang, W. Li, W. H. Dong, W. L. Mei and H. F. Dai, Phytochem. Lett., 2016, 17, 94-99.

38 W. Li, G. Liao, W. H. Dong, F. D. Kong, P. Wang, H. Wang, W. L. Mei and H. F. Dai, Molecules, 2016, 21, 274.

39 M. Ishihara, T. Tsuneya and K. Uneyama, Phytochemistry, 1993, 33, 1147-1155.

40 S. P. Gunasekera, A. D. Kinghorn, G. A. Cordell and N. R. Farnsworth, J. Nat. Prod., 1981, 44, 569-572.

41 T. C. Jain and S. C. Bhattacharyya, Tetrahedron Lett., 1959, 1, 13-17.

42 W. L. Mei, Y. B. Zeng, J. Liu and H. F. Dai, J. Chin. Med. Mater., 2007, 30, 551-555.

43 H. M. Deng, H. Q. Tong and R. J. Zhou, West China J. Pharm. Sci., 2008.

44 P. Wetwitayaklung, N. Thavanapong and J. Charoenteeraboon, Silpakorn Univ. Sci. Technol. J., 2009, 3, 25-33.

45 M. N. I. Bhuiyan, J. Begum and M. N. H. Bhuiyan, Bangladesh J. Pharmacol., 2009, 4, 24-28.

46 H. N. Wang, W. H. Dong, S. Z. Huang, W. Li, F. D. Kong, H. Wang, J. Wang, W. L. Mei and H. F. Dai, Fitoterapia, 2016, 114, 7-11.

47 K. X. Kang, H. F. Dai, P. Wang, F. D. Kong, L. M. Zhou, W. H. Dong, G. P. Zhu and W. L. Mei, Chin. Tradit. Herb. Drugs, 2017, 4601-4607.

48 T. D. Kuang, H. Q. Chen, W. Li, J. L. Yang, L. M. Zhou, C. H. Cai, W. H. Dong, W. L. Mei and H. F. Dai, China J. Chin. Mater. Med., 2017, 4618-4623.

49 W. Li, C. H. Cai, Z. K. Guo, H. Wang, W. J. Zuo, W. H. Dong, W. L. Mei and H. F. Dai, Fitoterapia, 2015, 100, 44-49.

50 B. Wu, J. G. Lee, C. J. Lim, S. D. Jia, S. W. Kwon, G. S. Hwang and J. H. Park, Helv. Chim. Acta, 2012, 4, 636-642.

51 S. L. Wang, Y. C. Tsai, S. L. Fu, M. J. Cheng, M. I. Chung and J. J. Chen, Molecules, 2018, 23, 289.

52 L. D. Lin and S. Y. Qi, Chin. Tradit. Herbal Drugs, 2000.

$53 \mathrm{H}$. Takemoto, M. Ito, T. Shiraki, T. Yagura and G. Honda, J. Nat. Med., 2008, 62, 41-46.

54 J. Pornpunyapat, P. Chetpattananondh and C. Tongurai, Bangladesh J. Pharmacol., 2011, 6, 18-24.

55 M. Ishihara, T. Tsuneya and K. Uneyama, Phytochemistry, 1991, 30, 3343-3347.

56 M. Ishihara, Y. Masatsugu and K. Uneyama, Tetrahedron, 1992, 48, 10265-10276.

57 D. L. Yang, W. Li, W. H. Dong, J. Wang, W. L. Mei and H. F. Dai, Fitoterapia, 2016, 112, 191-196. 
58 L. Yang, L. R. Qiao, D. Xie, J. G. Dai and S. X. Guo, China J. Chin. Mater. Med., 2012, 37, 1973.

59 L. Yang, L. R. Qiao, J. J. Zhang, J. G. Dai and S. X. Guo, J. Asian Nat. Prod. Res., 2012, 14, 1054-1058.

60 J. Liu, Master thesis, Hainan University, 2008.

61 C. T. Ma, T. Eom, E. Cho, B. Wu, T. R. Kim, K. B. Oh, S. B. Han, S. W. Kwon and J. H. Park, J. Nat. Prod., 2017, 80, 3043-3048.

62 P. Pant and R. P. Rastogi, Phytochemistry, 1980, 19, 18691870.

63 C. L. Miao, B. T. Sun, L. P. Luo, D. X. Liu and B. Y. Yang, Food Sci., 2010, 30, 215-217.

64 L. Yang, L. R. Qiao, J. J. Zhang, J. G. Dai and S. X. Guo, J. Asian Nat. Prod. Res., 2012, 14, 1054-1058.

65 J. S. Yang, Y. L. Wang and Y. L. Su, Acta Pharmacol. Sin., 1989, 24, 678-683.

66 J. S. Yang, Y. L. Wang and Y. L. Su, Acta Pharmacol. Sin., 1990, 25, 186-190.

67 J. M. Liu, Y. H. Gao, H. H. Xu and H. J. Chen, Chin. Tradit. Herb. Drugs, 2006, 325-327.

68 J. M. Liu, Y. H. Gao, H. H. Xu and Z. Q. Xu, Chin. Tradit. Herb. Drugs, 2007, 1138-1140.

69 J. Liu, J. Wu, Y. X. Zhao, Y. Y. Deng, W. L. Mei and H. F. Dai, Chin. Chem. Lett., 2008, 19, 934-936.

70 H. F. Dai, J. Liu, Y. B. Zeng, Z. Han, H. Wang and W. L. Mei, Molecules, 2009, 14, 5165-5168.

71 H. F. Dai, J. Liu, Z. Han, Y. B. Zeng, H. Wang and W. L. Mei, J. Asian Nat. Prod. Res., 2010, 12, 134-137.

72 T. Yagura, N. Shibayama, M. Ito, F. Kiuchi and G. Honda, Tetrahedron Lett., 2005, 46, 4395-4398.

73 L. Yang, L. R. Qiao, D. Xie, Y. H. Yuan, N. H. Chen, J. G. Dai and S. X. Guo, Phytochemistry, 2012, 76, 92-97.

74 Y. H. Gao, J. M. Liu, H. X. Lu and Z. X. Wei, Helv. Chim. Acta, 2012, 95, 951-954.

75 D. Chen, Z. R. Xu, X. Y. Chai, K. W. Zeng, Y. Jia, D. Bi, Z. Z. Ma and P. F. Tu, Eur. J. Org. Chem., 2012, 2012, 5389-5397.

76 D. L. Yang, W. L. Mei, Y. B. Zeng, Z. K. Guo, Y. X. Zhao, H. Wang, W. J. Zuo, W. H. Dong, Q. H. Wang and H. F. Dai, Planta Med., 2013, 79, 1329-1334.

77 H. Shao, F. D. Kong, H. Wang, W. L. Mei and H. F. Dai, J. Asian Nat. Prod. Res., 2017, 19, 935-940.

78 H. Shao, W. L. Mei, F. D. Kong, W. H. Dong, W. Li, G. P. Zhu and H. F. Dai, J. Asian Nat. Prod. Res., 2017, 19, 42-46.

79 W. Li, C. H. Cai, W. H. Dong, Z. K. Guo, H. Wang, W. L. Mei and H. F. Dai, Fitoterapia, 2014, 98, 117-123.

80 G. Liao, W. L. Mei, W. H. Dong, W. Li, P. Wang, F. D. Kong, C. J. Gai, X. Q. Song and H. F. Dai, Fitoterapia, 2016, 110, 3843.

81 G. Liao, W. L. Mei, F. D. Kong, W. Li, J. Z. Yuan and H. F. Dai, Phytochemistry, 2017, 139, 98-108.

82 P. Xiang, W. L. Mei, H. Q. Chen, F. D. Kong, H. Wang, G. Liao, L. M. Zhou and H. F. Dai, Fitoterapia, 2017, 120, 61-66.
83 Y. Y. Liu, D. L. Chen, J. H. Wei, J. Feng, Z. Zhang, Y. Yang and W. Zheng, Molecules, 2016, 21, 1433.

84 H. X. Huo, Y. F. Gu, H. Sun, Y. F. Zhang, W. J. Liu, Z. X. Zhu, S. P. Shi, Y. L. Song, H. W. Jin, Y. F. Zhao, P. F. Tu and J. Li, Fitoterapia, 2017, 118, 49-55.

85 H. X. Huo, Z. X. Zhu, Y. L. Song, S. P. Shi, J. Sun, H. Sun, Y. F. Zhao, J. Zheng, D. Ferreira, J. K. Zjawiony, P. F. Tu and J. Li, J. Nat. Prod., 2018, 81, 543-553.

86 Y. Shimada, T. Tominaga, T. Konishi and S. Kiyosawa, Chem. Pharm. Bull., 1982, 30, 3791-3795.

87 T. Konishi, A. Sugimoto, S. Kiyosawa and Y. Fujiwara, Chem. Pharm. Bull., 1992, 40, 778-779.

88 Y. Shimada, T. Konishi, S. Kiyosawa, M. Nishi, K. Miyahara and T. Kawasaki, Chem. Pharm. Bull., 1986, 34, 2766-2773.

89 Y. Shimada, T. Konishi and S. Kiyosawa, Chem. Pharm. Bull., 1986, 34, 3033-3037.

90 T. Nakanishi, A. Inada, M. Nishi, E. Yamagata and K. Yoneda, J. Nat. Prod., 2004, 49, 1106-1108.

91 K. Iwagoe, T. Konishi, S. Kiyosawa, Y. Shimada, K. Miyahara and T. Kawasaki, Chem. Pharm. Bull., 1988, 36, 2417-2422.

92 K. Iwagoe, T. Kakae, T. Konishi, S. Kiyosawa, Y. Fujiwara, Y. Shimada, K. Miyahara and T. Kawasaki, Chem. Pharm. Bull., 1989, 37, 124-128.

93 K. Iwagoe, S. Kodama, T. Konishi, S. Kiyosawa, Y. Fujiwara and Y. Shimada, Chem. Pharm. Bull., 1987, 35, 4680-4682.

94 T. Konishi, S. Kiyosawa, Y. Shimada, K. Miyahara and T. Kawasaki, Chem. Pharm. Bull., 2008, 37, 1428-1430.

95 T. Kornshi, K. Iwagoe, A. Sugimoto, S. Kiyosawa, Y. Fujiwara and Y. Shimada, Chem. Pharm. Bull., 1991, 39, 207-209.

96 T. Konishi, K. Iwagoe, S. Kiyosawa and Y. Fujiwara, Phytochemistry, 1989, 28, 3548-3550.

97 T. Konishi, K. Iwagoe, S. Kiyosawa and Y. Fujiwara, Chem. Pharm. Bull., 1991, 39, 1869-1870.

98 H. Z. Alkhathlan, H. M. Al-Hazimi, F. S. Al-Dhalaan and A. A. Mousa, Nat. Prod. Res., 2005, 19, 367-372.

99 T. Konishi, T. Konoshima, Y. Shimada and S. Kiyosawa, Chem. Pharm. Bull., 2002, 50, 419.

100 Y. Yang, W. L. Mei, F. D. Kong, H. Q. Chen, W. Li, Z. B. Chen and H. F. Dai, Fitoterapia, 2017, 119, 20-25.

101 Y. Yang, H. Q. Chen, F. D. Kong, L. M. Zhou, W. Li, W. H. Dong, Z. B. Chen, W. L. Mei and H. F. Dai, Phytochemistry, 2018, 145, 207-213.

102 T. Sugiyama, Y. Narukawa, S. Shibata, R. Masui and F. Kiuchi, J. Nat. Med., 2018, 1-8.

103 H. N. Wang, W. L. Mei, W. H. Dong, F. D. Kong, W. Li, J. Z. Yuan and H. F. Dai, J. Asian Nat. Prod. Res., 2018, 20, 122-127.

104 T. Yagura, M. Ito, F. Kiuchi, G. Honda and Y. Shimada, Chem. Pharm. Bull., 2003, 51, 560-564.

105 K. Hashimoto, S. Nakahara, T. Inoue, Y. Sumida, M. Takahashi and Y. Masada, Chem. Pharm. Bull., 1985, 33, 5088-5091. 